\title{
Complex Wavenumber Rossby Wave Ray Tracing
}

\section{Citation}

Shaman, Jeffrey, R. M. Samelson, and Eli Tziperman. 2012. “Complex Wavenumber Rossby Wave Ray Tracing." Journal of the Atmospheric Sciences 69 (7): 2112-33. https://doi.org/10.1175/jasd-11-0193.1.

\section{Permanent link}

http://nrs.harvard.edu/urn-3:HUL.InstRepos:41384982

\section{Terms of Use}

This article was downloaded from Harvard University's DASH repository, and is made available under the terms and conditions applicable to Other Posted Material, as set forth at http:// nrs.harvard.edu/urn-3:HUL.InstRepos:dash.current.terms-of-use\#LAA

\section{Share Your Story}

The Harvard community has made this article openly available.

Please share how this access benefits you. Submit a story.

\section{Accessibility}




\title{
Complex Wavenumber Rossby Wave Ray Tracing
}

\author{
JEFFREY SHAMAN \\ Department of Environmental Health Sciences, Columbia University, New York, New York \\ R. M. SAMELSON \\ College of Oceanic and Atmospheric Sciences, Oregon State University, Corvallis, Oregon \\ ELI TZIPERMAN \\ Department of Earth and Planetary Sciences, and School of Engineering and Applied Sciences, \\ Harvard University, Cambridge, Massachusetts
}

(Manuscript received 20 July 2011, in final form 5 March 2012)

\begin{abstract}
This paper presents a methodology for performing complex wavenumber ray tracing in which both wave trajectory and amplitude are calculated. This ray-tracing framework is first derived using a scaling in which the imaginary wavenumber component is assumed to be much smaller than the real wavenumber component. The approach, based on perturbation methods, is strictly valid when this scaling condition is met. The framework is then used to trace stationary barotropic Rossby waves in a number of settings. First, ray-traced Rossby wave amplitude is validated in a simple, idealized system for which exact solutions can be calculated. Complex wavenumber ray tracing is then applied to both solid-body rotation on a sphere and observed climatological upper-tropospheric fields. These ray-tracing solutions are compared with similarly forced solutions of the linearized barotropic vorticity equation (LBVE). Both real and complex wavenumber ray tracings follow trajectories matched by LBVE solutions. Complex wavenumber ray tracings on observed two-dimensional zonally asymmetric atmospheric fields are found to follow trajectories distinct from real wavenumber Rossby waves. For example, complex wavenumber ray tracings initiated over the eastern equatorial Pacific Ocean during boreal summer propagate northward and northeastward into the subtropics over the Atlantic Ocean, as well as southeastward into the Southern Hemisphere. Similarly initiated real wavenumber ray tracings remain within the deep tropics and propagate westward. These complex wavenumber Rossby wave trajectories and ray amplitudes are generally consistent with LBVE solutions, which indicates this methodology can identify Rossby wave effects distinct from traditional real wavenumber tracings.
\end{abstract}

\section{Introduction}

Ray tracing is often used to explore the propagation of Rossby waves with stationary or near-stationary phase speeds. These ray trajectories indicate how information is communicated through the atmosphere over large distances, as well as the time scales over which this information is conveyed. Rossby wave ray tracing has provided insight into the atmospheric response to steady

Corresponding author address: Jeffrey Shaman, Department of Environmental Health Sciences, Mailman School of Public Health, Columbia University, 722 West 168th Street, Rosenfield Building, Room 1104C, New York, NY 10032.

E-mail: j1s106@columbia.edu thermal and orographic forcing (Hoskins and Karoly 1981), the response to low-frequency forcing ( $\mathrm{Li}$ and Nathan 1994), tropical-extratropical interactions (Hoskins and Karoly 1981), interactions within the extratropics (Wang et al. 2007), and propagation within the tropics of easterly waves (Sobel and Bretherton 1999).

Heretofore, investigations have been restricted to Rossby waves with real wavenumbers. However, thermal or orographic forcing of the atmosphere will in theory produce large-scale waves with both complex and real wavenumbers. Rossby waves with complex wavenumbers will possess amplitudes that are modified, as they propagate, by the imaginary component of their wavenumber. Previous work has suggested that such modulated waves, given either a slowly decaying amplitude 
or growth through instability or interaction with the mean flow, may persist and be of importance for the communication of information over large distances within the atmosphere (Dickinson and Clare 1973).

Complex wavenumber ray tracing (CRT) has been used in other fields to describe phenomena such as caustic shadows (Kravtsov 2005), radio wave propagation and attenuation in the ionosphere (Budden and Terry 1970; Jones 1970), the diffraction of Gaussian beams (Keller and Streifer 1962), and the excitation of surface waves (Keller and Karal 1960). The utility of CRT lies in its ability, at times, to represent separate additional behaviors not captured by real wavenumber ray tracing (RRT). In particular, in situations where CRT and RRT behaviors diverge, CRT and RRT together provide a more complete description of wave response to a given forcing than either CRT or RRT alone. Alternatively, one may consider CRT as inclusive of RRT; that is, RRT is merely CRT where the imaginary component of the wavenumber equals zero (i.e., $\mathbf{k}_{i}=0$ ). From this perspective, CRT provides a more comprehensive set of wave packet solutions for a given forcing.

A number of approaches have been proposed and developed for carrying out CRT (Brillouin 1914; Sommerfeld 1914; Censor and Gavan 1989; Muschietti and Dum 1993; Kravtsov and Orlov 1999). These approaches typically approximate the ray equations for complex waves, as CRT of the full ray equations produces movement into complex space, which can be challenging to represent and interpret. Here we present a new method for tracing both the trajectory and amplitude of complex wavenumber waves. This approach is developed using perturbation methods and is then applied to the tracing of complex wavenumber barotropic Rossby waves in a zonally asymmetric two-dimensional environment. The method is appealing because of its relative simplicity, the introduced scaling formalism that retains an appropriate additional term in the ray-tracing equations, and the fact that CRT solutions replicate solutions of the full wave equation not captured by RRT.

In section 2 we provide theoretical context for the CRT of wave trajectories using perturbation methods. We expressly derive equations for ray-tracing twodimensional stationary barotropic Rossby waves with complex wavenumbers in section 3 . In section 4 we apply this complex Rossby wave ray tracing to a simple, idealized environment and show that the amplitude derived through ray tracing matches exact analytic solutions for the wave amplitude. We then perform both CRT and RRT on a sphere in solid-body rotation and compare these tracings to forced solutions of the linearized barotropic vorticity equation (LBVE) (section 5). CRT and RRT are then performed on realistic two-dimensional atmospheric flows and compared with forced solutions of the LBVE (section 6). Section 7 presents a comparison of our perturbation method with an alternate approach to CRT. Section 8 presents discussion.

\section{Ray tracing with complex wavenumbers}

\section{a. Ray equations}

The tracing of wave trajectories follows the general theory of ray tracing in an anisotropic medium (Whitham 1974; Lighthill 1978). Specifically, we consider a twodimensional, approximate plane wave solution with slowly varying amplitude, wavenumber, and position of the form $A \exp \left[i_{\phi}(x, y, t)\right]$, where $A$ is the wave amplitude, and the wave phase $\phi$ can be expressed locally as

$$
\phi(x, y, t) \approx k x+l y-\omega t,
$$

where $\omega(x, y, t)=-\partial \phi / \partial t$ is the slowly varying frequency, and $k(x, y, t)=\partial \phi / \partial x$ and $l(x, y, t)=\partial \phi / \partial y$ are slowly varying wavenumbers in the zonal and meridional directions, respectively. Per Wentzel-KramersBrillouin-Jeffries (WKBJ) theory, the length scale of the wave disturbance is short (i.e., there is rapid phase variation; Bender and Orszag 1978). Equality of the mixed partial derivatives then implies that the changes in $k$ and $l$ must satisfy

$$
\begin{aligned}
& \frac{d k}{d t}=-\frac{\partial \omega}{\partial x}, \\
& \frac{d l}{d t}=-\frac{\partial \omega}{\partial y},
\end{aligned}
$$

along wave rays defined by

$$
\begin{aligned}
& \frac{d x}{d t}=u_{g}=\frac{\partial \omega}{\partial k}, \\
& \frac{d y}{d t}=v_{g}=\frac{\partial \omega}{\partial l},
\end{aligned}
$$

where the group velocity vector $\mathbf{c}_{g}=\left(u_{g}, v_{g}\right)$ and $d / d t=$ $\partial / \partial t+\mathbf{c}_{g} \cdot \nabla$. With a dispersion relation $\omega_{0}=\omega(k, l ; x, y)$ determined from the local dynamics, Eqs. (2.2) and (2.3) provide a complete set of ODEs for $(x, y, k, l)$ from which the propagation of wave energy along a ray may be traced.

Typically, to perform stationary wave tracing, rays are initiated at some location $(x, y)$ with a prescribed integer zonal wavenumber $k$, and the initial meridional wavenumber $l$ is then solved for using the dispersion relation [i.e., $\omega(k, l, x, y)=\omega_{0}=0$ ]. Depending on the dispersion relation this procedure may yield multiple initial values 
for $l$, some of which may be complex. Ray tracing is usually only performed for real initial meridional wavenumber solutions; however, waves with complex dispersion relations, complex wavenumbers, and complex group velocities have been analyzed in fields such as geometric optics (Keller and Streifer 1962; Kravtsov 2005) and plasma physics (Budden and Terry 1970; Jones 1970) and have been used in geophysical fluid dynamics to describe convective and absolute instabilities of baroclinic eddies (Merkine 1977; Merkine and Shafranek 1980).

Muschietti and Dum (1993) and Sonnenschein et al. (1998) examined the propagation of waves in dissipative media, where the solution is also decaying, as it may be in dispersive media such as the inviscid rotating atmosphere considered here. Their approach was to let wavenumbers vary in the complex domain but restrict variations of the ray coordinates to real coordinate space by considering only the real part of the group velocity. This approach, based on saddle point analysis, is similar to the handling suggested by Sommerfeld (1914) and Brillouin (1914) for light traveling in absorptive medium, as well as the analysis of Merkine and Shafranek (1980) for the temporal and spatial evolution of unstable baroclinic disturbances.

\section{b. Implications and interpretation of complex wavenumber waves}

Before proceeding with our analysis, some reflection is warranted to consider what exactly a complex wavenumber wave constitutes. For these waves, the ray Eqs. (2.3) produce complex group velocities and, consequently, excursions of the rays into complex space. Many investigators have grappled with the physical interpretation of CRT, complex group velocity, and movement in complex space (Brillouin 1914; Connor and Felsen 1974; Censor and Gavan 1989; Muschietti and Dum 1993; Poli et al. 2001). These issues of interpretation, which are not generally resolved or agreed upon, have, perhaps, led to an underuse of CRT.

Kravtsov et al. (1999) defend CRT and discuss the difficulty of interpreting complex rays:

Although complex rays have been shown to be useful in the analysis of a variety of wave problems, their apparent intangibility has meant that they have sometimes been viewed negatively. Ironically, a moment's reflection shows that real rays have a similarly tenuous connection to the physical world; both real and complex rays are no more than convenient analytical frameworks for studying wave phenomena (p. 5).

Ultimately, the connection of complex wavenumber and group velocity waves to the real world is still a subject of debate (Censor and Gavan 1989; Kravtsov et al. 1999; Poli et al. 2001). Their interpretation and utility depends on the physical phenomenon being studied and the approximations used to estimate the complex wave behavior.

Censor and Gavan (1989) argue that movement into complex space obviates any notion of a real group velocity, representing wave packet and energy propagation, and that only the signal of the waves holding to the real plane can sensibly be considered part of a wave packet with an understandable, perceivable group velocity. Budden and Terry (1970) suggest that a complex ray is a Riemann surface in complex coordinate space. These authors, and others, have used CRT to describe the reflection and attenuation of radio waves in lossy media, such as the ionosphere (Budden and Terry 1970; Jones 1970).

Others have noted that complex waves can arise when an input signal or forcing has a complex amplitude dependence (Connor and Felsen 1974). CRT permits description of the spatial and temporal variations of the wave amplitude of the resulting waves, even in lossless media. Such propagation in lossless media of complex rays can describe evanescent wave phenomena such as the dark side of caustics, or growth due to spatial instabilities. Because complex wavenumbers are neither purely real nor purely imaginary, these waves are in essence mixed-propagating and evanescent phenomena.

A typical (real wavenumber) wave is one in which the real wave phase gradient is orthogonal to the imaginary part - that is, the wave packet decays in space orthogonally to the direction of propagation. For complex wavenumber waves, that angle between the real and imaginary phases is acute, so there is decay or growth in the direction of propagation (Kravtsov et al. 1999).

\section{c. A scaled approach to CRT}

For this work, we derive a framework for CRT using perturbation methods and a scaling of the imaginary and real components of the wavenumber. For a onedimensional system with real wavenumber $k$, Eq. (2.2a) simplifies to

$$
\frac{d k}{d t}=\frac{\partial k}{\partial t}+\mathbf{c}_{g} \frac{\partial k}{\partial x}=-\frac{\partial \omega}{\partial x} .
$$

Suppose now that $k$ is complex, such that $k=k_{r}+i k_{i}$. The dispersion relation and thus the group velocity will consequently be complex. The imaginary component of the wavenumber is readily interpretable as a modulation of plane wave amplitude [i.e., $A \exp \left(-k_{i} x\right)$ $\left.\exp \left(i k_{r} x-\omega t\right)\right]$; however, the complex group velocity leads to an imaginary trajectory, or characteristic, for which the physical interpretation is less clear. 
When $k$ is complex, but $x$ is required to remain real, the one-dimensional analog of Eq. (2.4) devolves into a pair of equations for the real and imaginary parts of $k$ :

$$
\begin{aligned}
& \frac{\partial k_{r}}{\partial t}+u_{g r} \frac{\partial k_{r}}{\partial x}=-\left.\frac{\partial \omega}{\partial x}\right|_{r}+u_{g i} \frac{\partial k_{i}}{\partial x}, \\
& \frac{\partial k_{i}}{\partial t}+u_{g r} \frac{\partial k_{i}}{\partial x}=-\left.\frac{\partial \omega}{\partial x}\right|_{i}-u_{g i} \frac{\partial k_{r}}{\partial x},
\end{aligned}
$$

where the subscripts $r$ and $i$ indicate real and imaginary parts, respectively. It can be seen by cross-differentiating the first and last terms of Eqs. (2.5a) and (2.5b) to eliminate $k_{i}$ in favor of $k_{r}$ that this pair of equations has an essentially elliptic character, and thus cannot be solved exactly along real characteristics.

Our goal is to find an approximate solution to Eq. (2.5) along real characteristics, despite the elliptic character of the full equations. Both the real and imaginary wavenumber equations (2.5) are expressed as an equality of the local change in time of the wavenumber plus the advection of the wavenumber by the real group velocity (lhs terms) with the local gradient of either the real [Eq. (2.5a)] or imaginary [Eq. (2.5b)] wave frequency plus the advection of the wavenumber by the imaginary group velocity (rhs terms). The first three terms of these equations are identical in form to Eq. (2.4) and represent the change in the wavenumber along the real characteristic. However, the last terms, which give the advection of the wavenumber by the imaginary group velocity, are new.

As we do not wish to follow ray paths that stray into complex space, we must eliminate or otherwise determine these last two terms. To do so we assume, per perturbation theory, that $k_{i}$ is $\mathcal{O}(\alpha)$ relative to $k_{r}$, where $\alpha \ll 1$, and that $k_{r}$ is $\mathcal{O}(1)$. Physically, this assumption implies a slow variation of the wave amplitude on a scale much longer than the wavelength. In addition, by assuming $k_{i} \ll k_{r}$, we are assuming that the angle between the real and imaginary phase gradients is nearly orthogonal.

The first three terms of Eq. (2.5a) are then $\mathcal{O}(1)$, but the last term is $\mathcal{O}\left(\alpha^{2}\right)$ and can be dropped, which reduces this equation to the form of Eq. (2.4). However, all four terms of Eq. (2.5b) are $\mathcal{O}(\alpha)$, so none can be eliminated. We therefore need to find an explicit way of describing the last term of this equation. To do so, we need an expression for $\partial k_{r} / \partial x$. Such an expression can be derived by taking the gradient of the $\mathcal{O}(1)$ terms in Eq. (2.5a).

\section{d. Wavenumber gradient equations}

We now develop an additional expression that represents the evolution of $\partial k_{r} / \partial x$ along the ray. Taking the partial derivative of Eq. (2.5a) with respect to $x$ after neglecting the $\mathcal{O}\left(\alpha^{2}\right)$ term and while holding $t$ fixed, we obtain

$$
\frac{\partial}{\partial t} \frac{\partial k_{r}}{\partial x}+u_{g r} \frac{\partial}{\partial x} \frac{\partial k_{r}}{\partial x}+\frac{\partial u_{g r}}{\partial x} \frac{\partial k_{r}}{\partial x}=-\left.\frac{\partial}{\partial x} \frac{\partial \omega}{\partial x}\right|_{r}
$$

and then make the additional approximations

$$
\frac{\partial u_{g r}}{\partial x}=\left.\left.\frac{\partial}{\partial x} \frac{\partial \omega}{\partial k}\right|_{r} \approx \frac{\partial^{2} \omega}{\partial x \partial k}\right|_{r}+\left.\frac{\partial^{2} \omega}{\partial k^{2}}\right|_{r} \frac{\partial k_{r}}{\partial x}
$$

and

$$
\left.\left.\frac{\partial}{\partial x} \frac{\partial \omega}{\partial x}\right|_{r} \approx \frac{\partial^{2} \omega}{\partial x \partial k}\right|_{r} \frac{\partial k_{r}}{\partial x}+\left.\frac{\partial^{2} \omega}{\partial x^{2}}\right|_{r},
$$

in which $\mathcal{O}\left(\alpha, \alpha^{2}\right)$ terms are neglected in the chain-rule expressions involving the complex $k(x)$. The first two terms of Eq. (2.6) are the derivative $d\left(\partial k_{r} / \partial x\right) / d t$ along the real ray $d x / d t=u_{g}$, while the rest can be written in terms of quantities evaluated on the ray.

Thus, our approximate complex wavenumber ray-tracing equations are the extended set

$$
\begin{aligned}
\frac{d k_{r}}{d t} & =-\left.\frac{\partial \omega}{\partial x}\right|_{r} \\
\frac{d k_{i}}{d t} & =-\left.\frac{\partial \omega}{\partial x}\right|_{i}+u_{g i} \frac{\partial k_{r}}{\partial x} \\
\frac{d}{d t}\left(\frac{\partial k_{r}}{\partial x}\right) & =-\left.\frac{\partial^{2} \omega}{\partial k^{2}}\right|_{r}\left|\frac{\partial k_{r}}{\partial x}\right|^{2}-\left.2 \frac{\partial^{2} \omega}{\partial k \partial x}\right|_{r} \frac{\partial k_{r}}{\partial x}-\left.\frac{\partial^{2} \omega}{\partial x^{2}}\right|_{r} \\
\frac{d x}{d t} & =u_{g r}=\left.\frac{\partial \omega}{\partial k}\right|_{r}
\end{aligned}
$$

In Eq. (2.9d), only the real part of the group velocity appears, giving a real ray trajectory, as the instantaneous value of the imaginary group velocity along the ray path is utilized in Eq. (2.9b) but does not directly contribute to determining the ray path. The critical approximation that allows the formulation (2.9) and solution along the real ray is the neglect of the last term in Eq. (2.5a) to obtain Eq. (2.9a).

\section{e. Two-dimensional form}

When $k$ and $l$ are complex, but $x$ and $y$ are required to remain real, the two-dimensional ray-tracing equations (2.2) devolve into a similar form as Eqs. (2.5):

$$
\begin{aligned}
\frac{d k_{r}}{d t} & =\frac{\partial k_{r}}{\partial t}+u_{g r} \frac{\partial k_{r}}{\partial x}+v_{g r} \frac{\partial k_{r}}{\partial y} \\
& =-\left.\frac{\partial \omega}{\partial x}\right|_{r}+u_{g i} \frac{\partial k_{i}}{\partial x}+v_{g i} \frac{\partial k_{i}}{\partial y},
\end{aligned}
$$

$\frac{d l_{r}}{d t}=\frac{\partial l_{r}}{\partial t}+u_{g r} \frac{\partial l_{r}}{\partial x}+v_{g r} \frac{\partial l_{r}}{\partial y}=-\left.\frac{\partial \omega}{\partial y}\right|_{r}+u_{g i} \frac{\partial l_{i}}{\partial x}+v_{g i} \frac{\partial l_{i}}{\partial y}$, 


$$
\begin{aligned}
& \frac{d k_{i}}{d t}=\frac{\partial k_{i}}{\partial t}+u_{g r} \frac{\partial k_{i}}{\partial x}+v_{g r} \frac{\partial k_{i}}{\partial y}=-\left.\frac{\partial \omega}{\partial x}\right|_{i}-u_{g i} \frac{\partial k_{r}}{\partial x}-v_{g i} \frac{\partial k_{r}}{\partial y}, \quad \frac{d}{d t}\left(\frac{\partial k_{r}}{\partial x}\right)=\frac{\partial}{\partial t}\left(\frac{\partial k_{r}}{\partial x}\right)+u_{g r} \frac{\partial}{\partial x}\left(\frac{\partial k_{r}}{\partial x}\right)+v_{g r} \frac{\partial}{\partial y}\left(\frac{\partial k_{r}}{\partial x}\right) \\
& \frac{d l_{i}}{d t}=\frac{\partial l_{i}}{\partial t}+u_{g r} \frac{\partial l_{i}}{\partial x}+v_{g r} \frac{\partial l_{i}}{\partial y}=-\left.\frac{\partial \omega}{\partial y}\right|_{i}-u_{g i} \frac{\partial l_{r}}{\partial x}-v_{g i} \frac{\partial l_{r}}{\partial y} . \\
& =-\left.2 \frac{\partial^{2} \omega}{\partial k \partial x}\right|_{r} \frac{\partial k_{r}}{\partial x}-\left.\frac{\partial^{2} \omega}{\partial k^{2}}\right|_{r}\left|\frac{\partial k_{r}}{\partial x}\right|^{2}-\left.2 \frac{\partial^{2} \omega}{\partial l \partial k}\right|_{r} \frac{\partial l_{r}}{\partial x} \frac{\partial k_{r}}{\partial x} \\
& -\left.2 \frac{\partial^{2} \omega}{\partial l \partial x}\right|_{r} \frac{\partial l_{r}}{\partial x}-\left.\frac{\partial^{2} \omega}{\partial l^{2}}\right|_{r}\left|\frac{\partial l_{r}}{\partial x}\right|^{2}-\left.\frac{\partial^{2} \omega}{\partial x^{2}}\right|_{r}
\end{aligned}
$$

As for the 1 D system, when $k_{i}$ and $l_{i}$ are $\mathcal{O}(\alpha)$ with $\alpha \ll 1$ we can neglect the last two $\mathcal{O}\left(\alpha^{2}\right)$ terms of Eqs. (2.10a) and $(2.10 \mathrm{~b})$, but we cannot drop the last two terms of Eqs. (2.10c) and (2.10d). As before, we derive explicit expressions for $\partial k_{r} / \partial x, \partial k_{r} / \partial y, \partial l_{r} / \partial x$, and $\partial l_{r} / \partial y$, using the $\mathcal{O}(1)$ terms of Eqs. (2.10a) and (2.10b):

$$
\begin{gathered}
\frac{d k_{r}}{d t}=\frac{\partial k_{r}}{\partial t}+\left.\frac{\partial \omega}{\partial k}\right|_{r} \frac{\partial k_{r}}{\partial x}+\left.\frac{\partial \omega}{\partial l}\right|_{r} \frac{\partial k_{r}}{\partial y}=-\left.\frac{\partial \omega}{\partial x}\right|_{r}, \\
\frac{d l_{r}}{d t}=\frac{\partial l_{r}}{\partial t}+\left.\frac{\partial \omega}{\partial k_{r}}\right|_{r} \frac{\partial l}{\partial x}+\left.\frac{\partial \omega}{\partial l}\right|_{r} \frac{\partial l_{r}}{\partial y}=-\left.\frac{\partial \omega}{\partial y}\right|_{r}
\end{gathered}
$$

Specifically, $\partial / \partial x$ of Eq. (2.11a) gives

where the relation $\partial k / \partial y=\partial^{2} \phi / \partial y \partial x=\partial l / \partial x$ has been used. Equation (2.12) is the approximate equation for the zonal gradient of $k$ along the ray [Eq. (2.3)]. All of the terms on the right-hand side of Eq. (2.12) can be evaluated along the ray.

Three more equations are needed to completely define the wavenumber gradients needed for the additional terms in the 2D imaginary wavenumber equations [Eqs. (2.10c) and (2.10d)]. These three remaining wavenumber gradient equations are derived similarly, by taking $\partial / \partial y$ of Eq. (2.11a) and $\partial / \partial x$ and $\partial / \partial y$ of Eq. (2.11b):

$$
\begin{aligned}
\frac{d}{d t}\left(\frac{\partial k_{r}}{\partial y}\right)= & -\left.\frac{\partial^{2} \omega}{\partial k \partial l}\right|_{r}\left(\frac{\partial l_{r}}{\partial y} \frac{\partial k_{r}}{\partial x}+\frac{\partial k_{r}}{\partial y} \frac{\partial k_{r}}{\partial y}\right)-\left.\frac{\partial^{2} \omega}{\partial k^{2}}\right|_{r} \frac{\partial k_{r}}{\partial y} \frac{\partial k_{r}}{\partial x}-\left.\frac{\partial^{2} \omega}{\partial l^{2}}\right|_{r} \frac{\partial l_{r}}{\partial y} \frac{\partial l_{r}}{\partial x}-\left.\frac{\partial^{2} \omega}{\partial y \partial x}\right|_{r}-\left.\frac{\partial^{2} \omega}{\partial k \partial x}\right|_{r} \frac{\partial k_{r}}{\partial y}-\left.\frac{\partial^{2} \omega}{\partial l \partial x}\right|_{r} \frac{\partial l_{r}}{\partial y} \\
& -\left.\frac{\partial^{2} \omega}{\partial k \partial y}\right|_{r} \frac{\partial k_{r}}{\partial x}-\left.\frac{\partial^{2} \omega}{\partial l \partial y}\right|_{r} \frac{\partial k_{r}}{\partial y}, \\
\frac{d}{d t}\left(\frac{\partial l_{r}}{\partial x}\right)= & -\left.\frac{\partial^{2} \omega}{\partial k \partial l}\right|_{r}\left(\frac{\partial l_{r}}{\partial x} \frac{\partial l_{r}}{\partial x}+\frac{\partial k_{r}}{\partial x} \frac{\partial l_{r}}{\partial y}\right)-\frac{\partial^{2} \omega}{\partial k^{2}}\left|\frac{\partial k_{r}}{\partial x} \frac{\partial l_{r}}{\partial x}-\frac{\partial^{2} \omega}{\partial l^{2}}\right|_{r} \frac{\partial l_{r}}{\partial x} \frac{\partial l_{r}}{\partial y}-\left.\frac{\partial^{2} \omega}{\partial x \partial y}\right|_{r}-\left.\frac{\partial^{2} \omega}{\partial k \partial y}\right|_{r} \frac{\partial k_{r}}{\partial x}-\left.\frac{\partial^{2} \omega}{\partial l \partial y}\right|_{r} \frac{\partial l_{r}}{\partial x} \\
& -\left.\frac{\partial^{2} \omega}{\partial k \partial x}\right|_{r} \frac{\partial l_{r}}{\partial x}-\left.\frac{\partial^{2} \omega}{\partial l \partial x}\right|_{r} \frac{\partial l_{r}}{\partial y}, \quad \text { and } \\
\frac{d}{d t}\left(\frac{\partial l_{r}}{\partial y}\right)= & -\left.2 \frac{\partial^{2} \omega}{\partial k \partial y}\right|_{r} \frac{\partial k_{r}}{\partial y}-\left.\frac{\partial^{2} \omega}{\partial k^{2}}\right|_{r} \frac{\partial k_{r}}{\partial y} \frac{\partial k_{r}}{\partial y}-2 \frac{\partial^{2} \omega}{\partial l \partial k}\left|\frac{\partial l_{r}}{\partial y} \frac{\partial k_{r}}{\partial y}-2 \frac{\partial^{2} \omega}{\partial l \partial y}\right|_{r} \frac{\partial l_{r}}{\partial y}-\left.\frac{\partial^{2} \omega}{\partial l^{2}}\right|_{r} \frac{\partial l_{r}}{\partial y} \frac{\partial l_{r}}{\partial y}-\left.\frac{\partial^{2} \omega}{\partial y^{2}}\right|_{r} .
\end{aligned}
$$

Equations (2.12)-(2.15) are the four wavenumber gradient equations needed to solve for the last two terms of Eqs. (2.10c) and (2.10d). The approximate ray-tracing equations for the two-dimensional complex waves are then Eqs. (2.11), (2.10c),(2.10d), (2.12)-(2.15), and (2.3). The dynamics of the waves will be controlled by the form of the dispersion relation $\omega_{0}=\omega(k, l, x, y)$, where $\omega_{0}$ is the wave frequency.

\section{f. Amplitude evolution for stationary complex wavenumber waves}

Wave amplitude along a ray is not constant but varies, per conservation of wave action density, with the convergence or divergence of rays within a ray tube or bundle, as described in Bretherton and Garrett (1969), Buehler (2009), and others. Conservation of wave action density, however, is distinct from the decay, or even growth, of a given ray due to a complex wavenumber, which can arise because of dissipation within the media or merely in the case where a wave has or is initiated with a complex amplitude dependence (Connor and Felsen 1974), as is the case here.

In the presence of dissipation, typically only decaying complex wavenumber waves are considered physically meaningful (Budden and Terry 1970; Jones 1970; Kravtsov 2005); however, in this study the ray-traced medium is to 
be treated as inviscid and complex relationships arise naturally from the dispersion relation. It is possible, though by no means definitive, that growing modes, if reasonably confined, represent spatial instabilities. For instance, CRT may have application in the ocean for describing the spatial instabilities that arise when waves cross the Gulf Stream front (Hogg 1988). Such changes in wave behavior in space (i.e., transitioning from a real or slowly decaying wave to a ray that grows or decays more precipitously) may be analogous to the use of CRT to describe light in and around caustics and caustic shadows (Kravtsov and Orlov 1999).

This conceptual framework is consistent with applications of CRT to geometric optics and radio waves in lossy media. In these applications the amplitude of the wave is explained partly by attenuation due to dissipation (i.e., the imaginary wavenumber-imposed decay of amplitude) and partly by conservation of wave action density (ray convergence) (Budden and Terry 1970; Kravtsov 2005). In this work, we are putting aside amplitude changes due to conservation of wave action density and only considering the changes that arise from the imaginary component of the wavenumber.

For stationary waves, which have $\omega_{0}=0$, the local amplitude variation of the wave packet near a point $\mathbf{x}_{0}=$ $\left(x_{0}, y_{0}\right)$ is described approximately by

$$
|A(\mathbf{x})|=\left|A\left(\mathbf{x}_{0}\right)\right| \exp \left[k_{i}(\epsilon \mathbf{x}) \cdot\left(\mathbf{x}-\mathbf{x}_{0}\right)\right], \quad \mathbf{x} \approx \mathbf{x}_{0},
$$

where $\mathbf{k}=(k, l), \mathbf{x}=(x, y)$, and $\epsilon \ll 1$. Note that $\epsilon$ is distinct from $\alpha$, which provides a relative scaling of $k_{i}$ and $k_{r}$; rather, $\epsilon$ is an arbitrarily small number such that $1 / \epsilon$ represents a large length scale. The local change in wave amplitude is then

$$
\begin{aligned}
\frac{d|A|}{d \mathbf{x}}(\mathbf{x}) & =\left[\mathbf{k}_{i}(\epsilon \mathbf{x})+\epsilon \frac{d \mathbf{k}_{i}}{d \mathbf{x}}(\epsilon \mathbf{x}) \cdot\left(\mathbf{x}-\mathbf{x}_{0}\right)\right]|A(\mathbf{x})|, \\
\mathbf{x} & \approx \mathbf{x}_{0} .
\end{aligned}
$$

As $\mathbf{x}_{0}$ is arbitrary in space and the term proportional to $\epsilon$ in Eq. (2.16) is arbitrarily small near $\mathbf{x}_{0}$, we may integrate Eq. (2.16) along the ray to obtain

$$
|A(\mathbf{x})| \approx|A(0)| \exp \left[\int_{0}^{\mathbf{x}} \mathbf{k}_{i}\left(\epsilon \mathbf{x}^{\prime}\right) \cdot d \mathbf{x}^{\prime}\right] .
$$

This expression may give either wave decay or wave growth, where the latter may indicate instability of the background flow.

In effect, we are exploring the case where $\epsilon \ll \alpha \ll 1$; that is, there are two different length scales, and the WKBJ length scale $1 / \epsilon$ is larger than the $1 / \alpha$ (complex wavenumber) length scale. Physically, this means that the exponential amplitude variation given by the imaginary wavenumber component is well defined (i.e., the variation looks exponential on the WKBJ local scales $L<1 / \epsilon$, where $k$ and $\omega$ are treated as constant). That is, we consider the case in which the exponential amplitude variation is assumed to dominate. Note that if $\alpha=0$ (i.e., $k_{i}=0$, etc.), then Eq. (2.17) degenerates to a constant amplitude on the WKBJ local scale.

\section{Barotropic Rossby waves}

Equations (2.10c), (2.10d), and (2.11)-(2.15), along with

$$
\begin{aligned}
& \frac{d x}{d t}=u_{g r}=\left.\frac{\partial \omega}{\partial k}\right|_{r} \text { and } \\
& \frac{d y}{d t}=v_{g r}=\left.\frac{\partial \omega}{\partial l}\right|_{r}
\end{aligned}
$$

provide the complete set of two-dimensional CRT equations for this approximation. For two-dimensional barotropic Rossby waves the dispersion relation is

$\omega(k, l, x, y)=\bar{u}_{M} k+\bar{v}_{M} l+\frac{l \partial \bar{q} / \partial x-k \partial \bar{q} / \partial y}{k^{2}+l^{2}}$,

where

$$
\left(\bar{u}_{M}, \bar{v}_{M}\right)=\frac{(\bar{u}, \bar{v})}{\cos \theta}
$$

is the Mercator projection of the time-mean zonal and meridional winds, $\theta$ is latitude,

$$
\bar{q}=2 \Omega \sin (\theta)+\nabla^{2} \bar{\psi}
$$

is the time-mean absolute vorticity, and $\Omega$ is the rotation rate of the earth.

Using this dispersion relation and Eq. (2.11) gives the following ray-tracing equations for the real parts of the wavenumbers:

$\frac{d k_{r}}{d t}=\operatorname{Re}\left(-k \frac{\partial \bar{u}_{M}}{\partial x}-l \frac{\partial \bar{v}_{M}}{\partial x}+\frac{k \partial^{2} \bar{q} / \partial x \partial y-l \partial^{2} \bar{q} / \partial x^{2}}{k^{2}+l^{2}}\right)$,

$$
\frac{d l_{r}}{d t}=\operatorname{Re}\left(-k \frac{\partial \bar{u}_{M}}{\partial y}-l \frac{\partial \bar{v}_{M}}{\partial y}+\frac{k \partial^{2} \bar{q} / \partial y^{2}-l \partial^{2} \bar{q} / \partial x \partial y}{k^{2}+l^{2}}\right)
$$

along the real rays [from Eq. (3.1)]: 


$$
\begin{aligned}
\frac{d x}{d t} & =u_{g r} \\
& =\operatorname{Re}\left(\frac{\partial \omega}{\partial k}\right) \\
& =\operatorname{Re}\left[\bar{u}_{M}+\frac{\left(k^{2}-l^{2}\right) \partial \bar{q} / \partial y-2 k l \partial \bar{q} / \partial x}{\left(k^{2}+l^{2}\right)^{2}}\right], \\
\frac{d y}{d t} & =v_{g r} \\
& =\operatorname{Re}\left(\frac{\partial \omega}{\partial l}\right) \\
& =\operatorname{Re}\left[\bar{v}_{M}+\frac{2 k l \partial \bar{q} / \partial y+\left(k^{2}-l^{2}\right) \partial \bar{q} / \partial x}{\left(k^{2}+l^{2}\right)^{2}}\right] .
\end{aligned}
$$

The imaginary group velocity $\mathbf{c}_{g i}$ is evaluated locally as

$$
\begin{aligned}
& u_{g i}=\operatorname{Im}\left(\frac{\partial \omega}{\partial k}\right)=\operatorname{Im}\left[\bar{u}_{M}+\frac{\left(k^{2}-l^{2}\right) \partial \bar{q} / \partial y-2 k l \partial \bar{q} / \partial x}{\left(k^{2}+l^{2}\right)^{2}}\right], \\
& v_{g i}=\operatorname{Im}\left(\frac{\partial \omega}{\partial l}\right)=\operatorname{Im}\left[\bar{v}_{M}+\frac{2 k l \partial \bar{q} / \partial y+\left(k^{2}-l^{2}\right) \partial \bar{q} / \partial x}{\left(k^{2}+l^{2}\right)^{2}}\right] .
\end{aligned}
$$

The expressions needed to piece together Eqs. (2.12)(2.15) are provided in appendix A. Using those component expressions we can form the ray-tracing equations for $\partial k_{r} / \partial x, \partial k_{r} / \partial y, \partial l_{r} / \partial x$, and $\partial l_{r} / \partial y$, and thus also Eqs. (2.10c) and (2.10d) for $k_{i}$ and $l_{i}$.

\section{Analytical example: Amplitude variation}

We next apply this two-dimensional complex wavenumber Rossby wave ray tracing to a simple, idealized setting for which an exact analytic solution of wave amplitude can also be calculated. We employ an infinite $\beta$ plane that for the region $0<y<Y$ additionally has bottom topography $\beta_{T}$. Thus, there are three regions: $y<0,0<y<Y$ and $y>Y$ (I, II, and III, respectively). Conditions are imposed such that the first and last regions (I and III) only support real Rossby waves; the middle section (region II), given a specific range of bottom topographies, creates a meridional gradient of potential vorticity $\beta+\beta_{T}$ that supports only evanescent wave modes, which are represented as complex wavenumber Rossby waves (Fig. 1).

Throughout the entire domain (regions I, II, and III), the zonal flow $\bar{u}_{M}$ is constant, the meridional flow $\bar{v}_{M}$ is zero, and a zonal gradient of absolute vorticity $\partial \bar{q} / \partial x$ is imposed as bottom topography and is constant for all

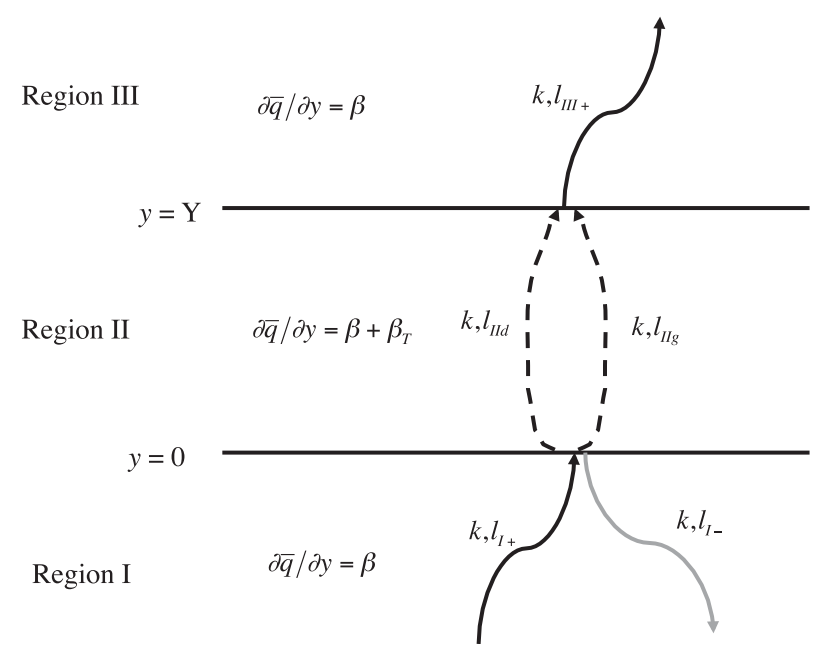

FIG. 1. Schematic of the infinite $\beta$ plane used for comparing raytraced and exact-amplitude solutions.

three regions. For continuity to be met at all time and all values of $x$, the wave frequency and zonal wavenumber must match at the two boundaries $(y=0$ and $y=Y)$, so $\omega$ and $k$ are constant everywhere. The same cannot be claimed for the meridional wavenumbers, due to the variation of $\beta$ in the $y$ direction. Thus, the solution for the wave streamfunction $\psi_{X}$ in region $X=\{\mathrm{I}, \mathrm{II}, \mathrm{III}\}$ has the form

$$
\psi_{X}=\sum_{j=1}^{J_{X}} A_{X_{j}} \exp \left[i\left(k x+l_{X_{j}} y-\omega t\right)\right]
$$

where $J_{X}$ is the number of wave modes in region $X$, and $\omega$ and $k$ are independent of the region.

For this system, regions I and III support two real wave modes for the prescribed $k, u, \beta, \partial \bar{q} / \partial x, v$, and $\omega_{0}$. The two waves possess real meridional wavenumber of opposite sign and thus propagate in opposite directions (one northward, one southward). For the problem we initiate a single, northward-propagating Rossby wave with amplitude 1 within region I. Upon reaching the $y=$ 0 bound two transmitted waves and a reflected wave are generated. The reflected wave is the southwardpropagating Rossby wave supported by region I.

For region II, two Rossby wave modes with complex conjugate meridional wavenumbers are supported. The imaginary part of the meridional wavenumber modulates the wave amplitude; one mode is decaying, the other is growing. (Note that the real wavenumber component produces wave propagation; the imaginary wavenumber component produces amplitude decay or growth.) By design, the solutions have positive real meridional wavenumber components, such that both waves propagate northward. Consequently, no reflection is supported when 
these waves reach the $y=Y$ boundary (given the prescribed $\bar{u}_{M}, \bar{v}_{M}, \beta, \beta_{T}, \partial \bar{q} / \partial x$, and $\left.\omega_{0}\right)$.

For region III, only the northward wave is spawned by incidence of the complex waves at $y=Y$. Thus at $y=0$, we have four waves to consider, the region I incident northward-propagating real wave, the region I reflected southward-propagating real wave, and the two region II northward-propagating complex waves (Fig. 1). These four waves satisfy two boundary conditions at $y=0$, discontinuity matching of the quasigeostrophic potential vorticity equation and continuity of pressure across the boundary. These boundary conditions are derived in appendix B.

At $y=Y$, we have only three waves to consider, the two northward-propagating region II complex waves and the region III northward-propagating real wave (Fig. 1). These three waves must satisfy the same two boundary conditions at $y=Y$. Thus, the system requires that

$$
\begin{gathered}
\psi_{\mathrm{I} n}+\psi_{\mathrm{I} s}=\psi_{\mathrm{II} g}+\psi_{\mathrm{II} d} \text { at } y=0 \\
\frac{\partial \psi_{\mathrm{I} n}}{\partial y}+\gamma \psi_{\mathrm{I} n}+\frac{\partial \psi_{\mathrm{I} s}}{\partial y}+\gamma \psi_{\mathrm{I} s}=\frac{\partial \psi_{\mathrm{IIg}}}{\partial y}+\gamma \psi_{\mathrm{II} g} \\
+\frac{\partial \psi_{\mathrm{II} d}}{\partial y}+\gamma \psi_{\mathrm{II} d} \text { at } y=0 \\
\psi_{\mathrm{III}}=\psi_{\mathrm{II} g}+\psi_{\mathrm{II} d} \text { at } y=Y
\end{gathered}
$$$$
\frac{\partial \psi_{\mathrm{III}}}{\partial y}+\gamma \psi_{\mathrm{III}}=\frac{\partial \psi_{\mathrm{II} g}}{\partial y}+\gamma \psi_{\mathrm{II} g}+\frac{\partial \psi_{\mathrm{II} d}}{\partial y}+\gamma \psi_{\mathrm{II} d} \quad \text { at } \quad y=Y,
$$

where the subscripts In, Is, II $g$, II $d$, and III denote the region I northward-, region I southward-, region II growing, region II decaying, and region III northwardpropagating Rossby waves, respectively, and $\gamma=\bar{q}_{x} / u_{M} k$.

The term $\psi_{\mathrm{I} n}$ is prescribed with amplitude 1 . We solve for the four other waves given the above system [Eq. (4.2)]. The solutions (see appendix B) give the amplitude of each wave, which varies for a given background and region I zonal wavenumber Rossby wave as a function of region II domain size.

We then compare this amplitude solution to a CRT of region II from $y=0$ to $y=Y$. Within region II the dispersion relation [Eq. (3.2)] reduces to

$$
\omega=\bar{u}_{M} k+\frac{l \partial \bar{q} / \partial x-k \partial \bar{q} / \partial y}{k^{2}+l^{2}} ;
$$

the ray-tracing equations are

$$
\frac{d k}{d t}=-\frac{\partial \omega}{\partial x}=0 \quad \text { and }
$$

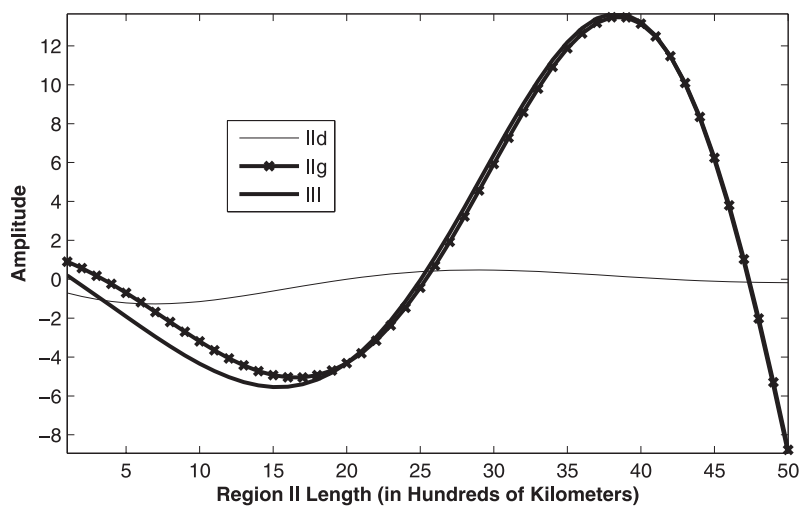

FIG. 2. Wave height solutions for Rossby waves on infinite $\beta$ plane, as described in text. Solutions are shown for the three Rossby waves at $y=Y$ as a function of region II domain size.

$$
\frac{d l}{d t}=-\frac{\partial \omega}{\partial y}=0
$$

and

$$
\begin{aligned}
\frac{d x}{d t} & =u_{g}=\operatorname{Re}\left(\frac{\partial \omega}{\partial k}\right) \\
& =\operatorname{Re}\left[\bar{u}_{M}+\frac{\left(k^{2}-l^{2}\right) \partial \bar{q} / \partial y-2 k l \partial \bar{q} / \partial x}{\left(k^{2}+l^{2}\right)^{2}}\right] \text { and }
\end{aligned}
$$

$\frac{d y}{d t}=v_{g}=\operatorname{Re}\left(\frac{\partial \omega}{\partial l}\right)=\operatorname{Re}\left[\frac{2 k l \partial \bar{q} / \partial y+\left(k^{2}-l^{2}\right) \partial \bar{q} / \partial x}{\left(k^{2}+l^{2}\right)^{2}}\right]$,

respectively; also, the evolution of the wave amplitude along the ray is described by

$$
\frac{d A}{d y}=-l_{i} A
$$

Equation (4.4) makes clear that within region II there is no change of the Rossby wave wavenumbers, either real or complex; rather, this simple analytic system enables comparison of calculated and ray traced complex wavenumber Rossby wave amplitudes. Furthermore, because Eq. (4.3) is quadratic in $l$, within region II, $l_{\mathrm{II}}$ and $l_{\mathrm{II}_{d}}$ are complex conjugates. Note that the two solution methods are distinct: the ray tracing integrates an initialvalue problem within region II; the analytic solution solves a boundary value problem for all three regions.

Figure 2 shows how wave height solutions vary as a function of $Y$ for $\bar{u}_{M}=20 \mathrm{~m} \mathrm{~s}^{-1}, \bar{v}_{M}=0 \mathrm{~m} \mathrm{~s}^{-1}, \beta=2 \times$ $10^{-11} \mathrm{~s}^{-1} \mathrm{~m}^{-1}, \beta+\beta_{T}=-3.26 \times 10^{-11} \mathrm{~s}^{-1} \mathrm{~m}^{-1}$, $\partial \bar{q} / \partial x=-4.5 \times 10^{-11} \mathrm{~s}^{-1} \mathrm{~m}^{-1}, \quad \omega_{0}=0$, and zonal wavenumber $k=7.85 \times 10^{-7} \mathrm{~m}^{-1}$. The wave height is the real part of the wave function, the amplitude times 


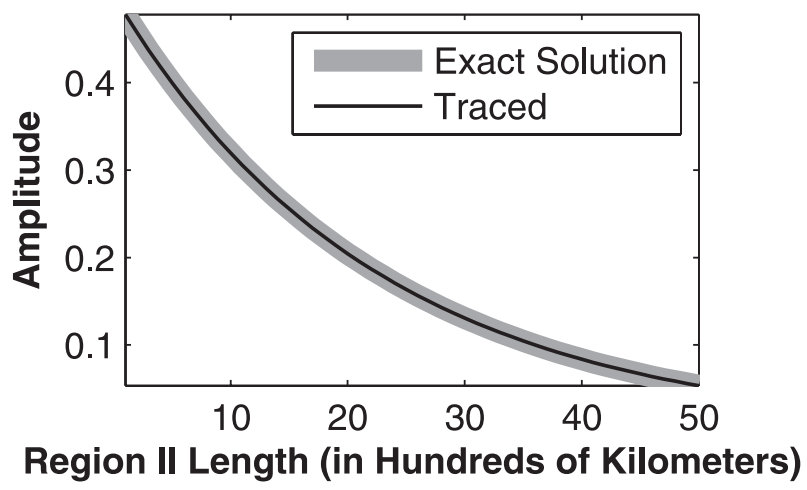

FIG. 3. Comparison of analytic and ray-traced amplitude solutions of region II decaying mode at $y=Y$ for a range of region II domain sizes. Solutions are for the same system as in Fig. $2: u=20 \mathrm{~m} \mathrm{~s}^{-1}, v=$ $0 \mathrm{~m} \mathrm{~s}^{-1}, \beta=2 \times 10^{-11} \mathrm{~s}^{-1} \mathrm{~m}^{-1}, \beta+\beta_{T}=-3.26 \times 10^{-11} \mathrm{~s}^{-1} \mathrm{~m}^{-1}$, $\partial \bar{q} / \partial x=-4.5 \times 10^{-11} \mathrm{~s}^{-1} \mathrm{~m}^{-1}$, and $k=5$. The thick gray line shows the analytic solution; the thin black line is the ray-traced solution.

the wave phase, e.g., $\operatorname{Re}\left(\psi_{\mathrm{II}}\right)=\operatorname{Re}\left[\sum A_{\mathrm{II} j} \exp \left(l_{\mathrm{II} j_{j}} y\right)\right.$ $\left.\operatorname{expi}\left(k x+l_{\mathrm{II}_{j_{r}}} y-\omega_{0} t\right)\right]$ in region II. Wave heights are shown at $y=Y$ as this quantity accounts for wave phase and is the quantity matched in the system solution at this location (see appendix B). At $y=Y$, the region III Rossby wave height closely matches the height of the region II growing mode.

Figure 3 shows the amplitude of the region II decaying mode at $y=Y$, as well as the amplitude of this mode calculated numerically via the CRT amplitude [Eq. (4.6)]. The two solutions match well, showing that CRT appropriately captures the modulation of wave amplitude, as well as wave propagation characteristics, which define this modulation. Growing solutions also match (not shown). This analysis was repeated for a number of backgrounds and initial zonal wavenumbers; in all cases the ray-tracing and analytic solutions matched.

\section{Solid-body rotation}

We next performed stationary barotropic Rossby wave ray tracing of both complex and real wavenumber waves on a variety of two-dimensional fields. Both real and complex ray-tracing trajectories were validated through comparison with forced solutions of the barotropic vorticity equation (LBVE) linearized about the same twodimensional fields (see appendix $\mathrm{C}$ for details).

We first experimented with solid-body rotation where $\bar{u}_{M}$ is constant, $\bar{v}_{M}=0$, and $\bar{q}_{x}=0$. Thus, the twodimensional dispersion relation reduces to

$$
\omega=\bar{u}_{M} k+\frac{-\bar{q}_{y} k}{k^{2}+l^{2}} .
$$

The ray-tracing equations are again Eqs. (2.10c) and (2.10d), (2.11)-(2.15), and (3.1). In this instance, the real wavenumber and group velocity equations are somewhat simplified to

$$
\begin{aligned}
& \frac{d k_{r}}{d t}=-\frac{\partial \omega}{\partial x}=0, \\
& \frac{d l_{r}}{d t}=-\frac{\partial \omega}{\partial y}=\frac{k \partial^{2} \bar{q} / \partial y^{2}}{k^{2}+l^{2}},
\end{aligned}
$$

and

$$
\begin{aligned}
& \frac{d x}{d t}=u_{g r}=\operatorname{Re}\left(\frac{\partial \omega}{\partial k}\right)=\operatorname{Re}\left[\bar{u}_{M}+\frac{\left(k^{2}-l^{2}\right) \partial \bar{q} / \partial y}{\left(k^{2}+l^{2}\right)^{2}}\right], \\
& \frac{d y}{d t}=v_{g r}=\operatorname{Re}\left(\frac{\partial \omega}{\partial l}\right)=\operatorname{Re}\left[\frac{2 k l \partial \bar{q} / \partial y}{\left(k^{2}+l^{2}\right)^{2}}\right]
\end{aligned}
$$

respectively. The evolution of the wave amplitude is described by Eq. (2.17). The imaginary wavenumber expression includes the additional terms seen in Eqs. (2.10c) and (2.10d).

We performed these solid-body rotation experiments using $\bar{u}_{M}=15 \mathrm{~m} \mathrm{~s}^{-1}$. At first, to insure that $\alpha \ll 1$ (i.e., $k_{i} \ll k_{r}$ and $l_{i} \ll l_{r}$ ), we initialized the complex wavenumber ray tracings with an apportioned complex zonal wavenumber (e.g., $k=5+0.01 i$ ), such that, given the background flow, the meridional wavenumber was similarly scaled among its real and imaginary components. Figure 4 presents the ray-tracing integrations for four real wavenumber stationary Rossby waves initiated with zonal wavenumber $k=5$ and four complex wavenumber stationary Rossby waves initiated with zonal wavenumber $k=5+0.01 i$. These RRTs and CRTs are pairwise initiated at the same locations and are superimposed on the steady solution of the LBVE model in response to forcing centered at $0^{\circ}, 180^{\circ}$. The trajectories of real and complex wavenumber rays initiated at the same location appear identical in Fig. 4; however, in fact, they do not match precisely. The small imaginary complex wavenumber introduces slight changes in amplitude and trajectory over the course of the ray integration.

These differences can be seen more clearly in plots of the evolution of the wavenumbers and amplitude of one real wavenumber stationary Rossby wave ray tracing and the corresponding complex wavenumber stationary Rossby wave ray tracing, initiated at the same location (Fig. 5). For this background, the ray tracing initiated with zonal wavenumber $k=5$ has a real initial meridional wavenumber. As both wavenumbers are real, there is no change in amplitude during the ray-tracing integration. The ray tracing initiated with zonal wavenumber 


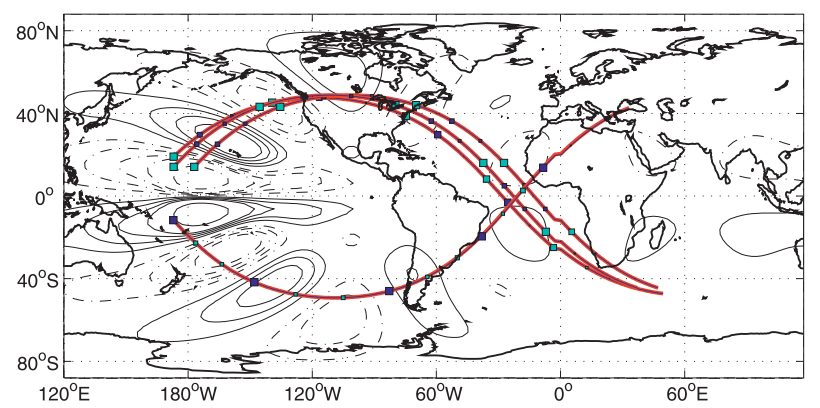

FIG. 4. LBVE steady vorticity solution to Gaussian amplitude divergent forcing centered at $0^{\circ}, 180^{\circ}$. Divergence maximizes in center at $-3 \times 10^{-6} \mathrm{~s}^{-1}$. The background is in solid-body rotation that maximizes at $15 \mathrm{~m} \mathrm{~s}^{-1}$ at the equator. Planetary rotation is as for Earth. Superimposed on this solution are ray tracings initiated within the forcing region with initial $k=5$ (blue lines) and $k=5+$ $0.01 i$ (red lines) initiated within the forcing region. All waves are integrated for 15 days. The complex and real ray tracings are indistinguishable, so square markers are additionally used to show the location of the real ray trajectory every $24 \mathrm{~h}$. These markers also indicate wave phase-dark blue is positive, light blue is negativeand marker size indicates phase magnitude. All rays begin at either maximal or minimal phase regardless of forcing location. Black contours show the LBVE solution with contour intervals of $0.5 \times$ $10^{-6}$ to $\pm 2 \times 10^{-6} \mathrm{~s}^{-1}$ and $2 \times 10^{-6} \mathrm{~s}^{-1}$ for larger magnitudes. Negative contours are dashed; the zero contour is omitted.

$k=5+0.01 i$, however, produces a slight decay of amplitude during integration; this small amplitude decay reflects the small magnitude of the imaginary wavenumbers. During the 15-day integration these imaginary wavenumbers remain several orders of magnitude smaller than the real wavenumbers, consistent with our assumption that $\alpha \ll 1$. Both the real and complex wavenumber trajectories match the steady solution of the LBVE model (Fig. 4).

A number of the CRTs initiated with $k=5+0.01 i$ behaved erratically and diverged from their real wavenumber counterparts (Figs. 6 and 7). This divergence occurred when the imaginary wavenumber component grew to magnitudes equal to that of the real wavenumber component (i.e., $\alpha \approx 1$ ). At that point, the wave trajectory would devolve to a primarily zonal propagation and the wave amplitude would shift radically. This erratic behavior occurred in the vicinity of the turning latitude where meridional propagation approaches zero. Near the turning latitude of a ray, $k_{r} / l_{r}$ is large, WKBJ assumptions break down, and even real wavenumber ray-tracing solutions are not strictly valid (Karoly and Hoskins 1982). However, similar erratic behavior does not arise for any of the RRTs, which propagate through one or more turning latitudes.

Indeed, the particular sensitivity of some of the CRTs near the turning latitude suggests that these ray tracings are more prone to erratic behavior, which likely arises from violation of the assumption that $\alpha \ll 1$ or from violation of both WKBJ scaling and $\alpha \ll 1$. Examination of the integrations of a number of these erratic ray tracings consistently revealed $l_{i}>l_{r}$ for successive steps in the region of the turning latitude, as well as unstable behavior in some of the terms used to determine $\partial k_{r} / \partial \mathbf{x}$, specifically those with $\left(k^{2}+l^{2}\right)^{3}$ in the denominator. These changes produced further growth of $l_{i}$ by several orders of magnitude while $l_{r}$ remained near zero.

As a consequence, the erratic and aphysical ray-tracing solutions of complex wavenumber waves should be dismissed from the point at which this erratic behavior arises. However, as will be shown in the next section we did find numerous wave tracings initiated on realistic twodimensional fields for which the $\alpha \ll 1$ assumption was violated but wave behavior remained reasonable and matched LBVE model solutions.

\section{Realistic two-dimensional atmospheric fields}

For the next set of experiments we again performed ray tracing and found forced solutions of the LBVE model using National Centers for Environmental Prediction (NCEP)-National Center for Atmospheric Research (NCAR) reanalysis July-September 1949-2010 300-hPa relative vorticity climatology (Kalnay et al. 1996) as the background. Gaussian divergence forcing was applied centered at $90^{\circ} \mathrm{W}$ and $5^{\circ} \mathrm{N}$ that extended $20^{\circ}$ to the east and west but only $5^{\circ}$ to the north and south of this center. This forcing mimics the position of precipitation anomalies associated with boreal summer El Niño events (Shaman and Tziperman 2007).

For ray tracing with this background, the full twodimensional dispersion relation [Eq. (3.2)] now applies. Note that the shear terms in the time-mean absolute vorticity are retained in full, as they are the same order of magnitude as $\beta$ for this background. For each ray initiation point and initial zonal wavenumber, there are now three initial meridional wavenumber solutions. Per Eq. (2.10c), the zonal wavenumber changes as the Rossby wave propagates such that a real zonal wavenumber becomes complex if the meridional wavenumber is complex. The Rossby wave amplitude is again modified by the imaginary components of both the zonal and meridional wavenumbers [Eq. (2.17)].

Figure 8 presents solutions for initial integer zonal wavenumbers $k=4-12$ at all sites within $0^{\circ}-10^{\circ} \mathrm{N}$ and $110^{\circ}-70^{\circ} \mathrm{W}$ superimposed on the steady vorticity solution of the LBVE model. The trajectories of the RRTs and CRTs clearly diverge. The real wavenumber stationary barotropic Rossby waves remain trapped within the equatorial waveguide and propagate westward, whereas 

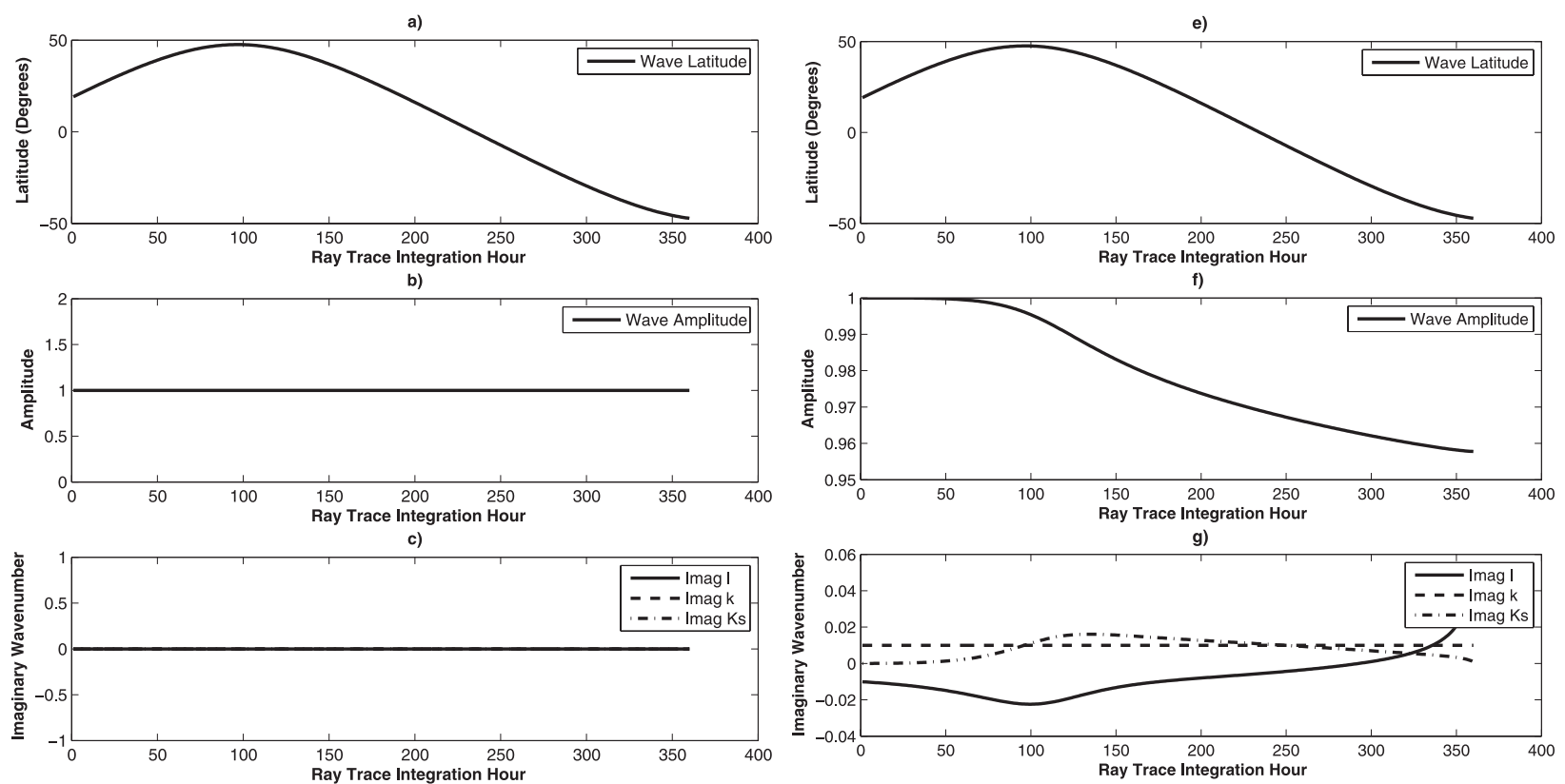

d)
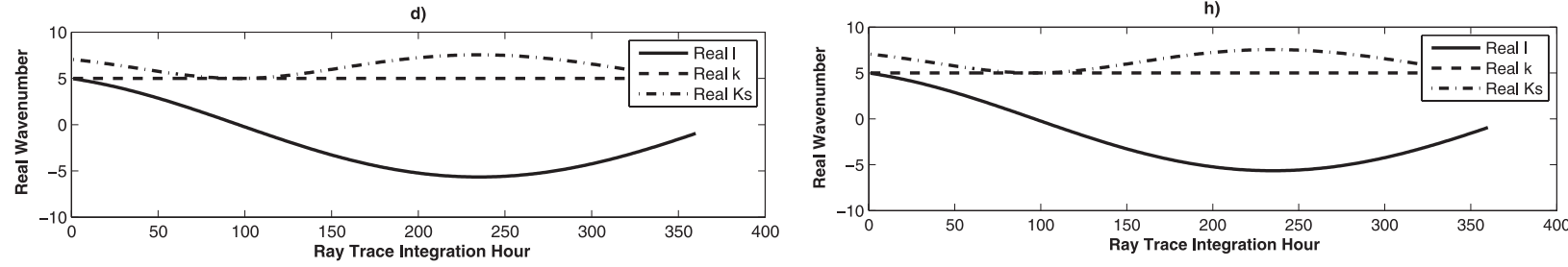

FIG. 5. Two examples of ray-tracing solutions initiated at the same location $\left(18.62^{\circ} \mathrm{N}, 172.5^{\circ} \mathrm{E}\right)$ on solid-body rotation with $u=$ $15 \mathrm{~m} \mathrm{~s}^{-1}$ at the equator, showing ray tracings with initial zonal wavenumber (a)-(d) $k=5$ and (e)-(h) $k=5+0.01 i$. Shown are (a),(e) wave latitude, (b),(f) wave amplitude, (c),(g) imaginary wavenumber parts, and (d),(h) real wavenumber parts during 15 days $(360 \mathrm{~h})$ of integration.

the complex wavenumber stationary barotropic Rossby waves typically escape the equatorial region and propagate into the subtropics of both the Northern and Southern Hemisphere.

Figure 9 shows separately the ray-tracing solutions for initial zonal wavenumbers $k=4-6$. Many of the complex wavenumber stationary barotropic Rossby wave ray tracings move along trajectories that fall into three general groupings. The first grouping propagates northward to the region of subtropical convergence to the north of the forcing area and then propagates westward. Some of these rays propagate farther north and then move northeastward over the continental United States. The second grouping moves northward or northeastward over the Caribbean. Some of these rays propagate eastnortheastward over Cuba and a few, by day 15, extend into the North African-Asian jet. The third grouping propagates southward to about $10^{\circ} \mathrm{S}$ and then moves eastward.

Rossby wave propagation is strongly affected by the background flow, in particular gradients of absolute vorticity and waveguide structures (Hoskins and Ambrizzi 1993). Figure 10 presents 1949-2010 July-September (JAS) 300-hPa climatology of the zonal wind; the meridional gradient of absolute vorticity $\beta_{M}$ per Karoly (1983),

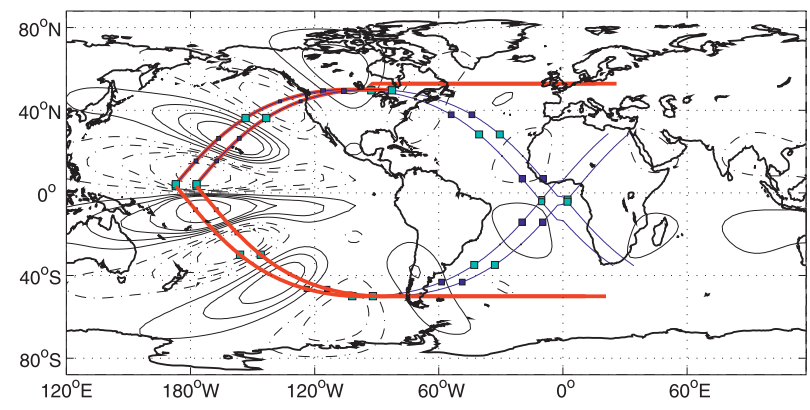

FIG. 6. As in Fig. 4, pairs of ray tracings initiated within the forcing region with initial $k=5$ (blue lines) and $k=5+0.01 i$ (red lines). For these tracings the complex wavenumber Rossby waves remain at a poleward latitude rather than turning equatorward with their real counterparts. 

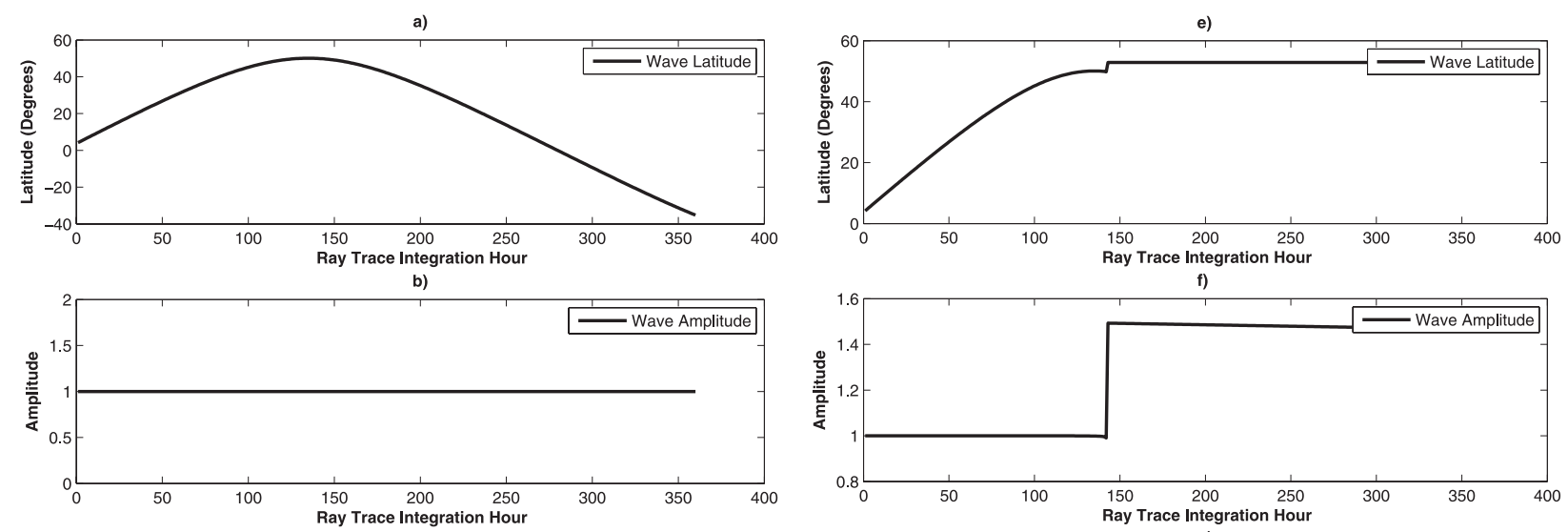

c)
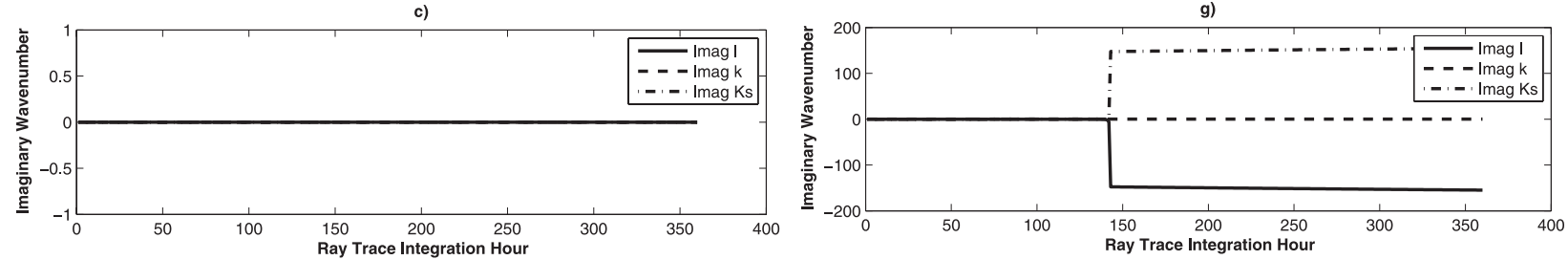

d)
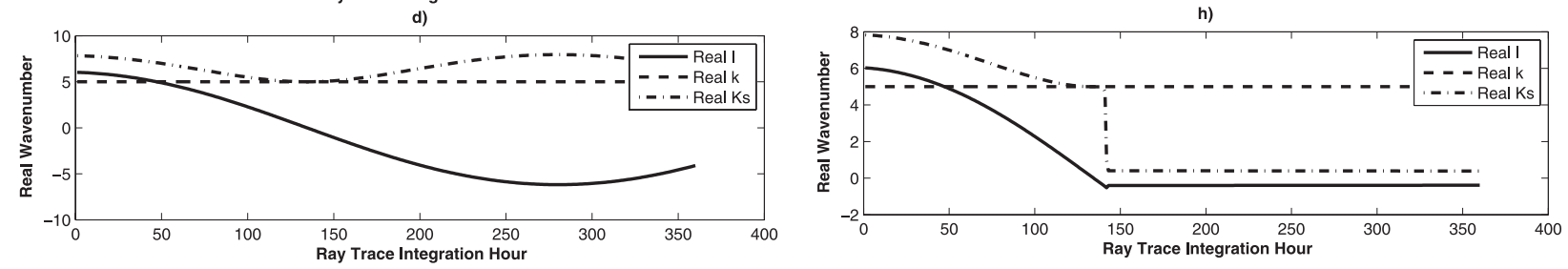

FIG. 7. Two examples of ray-tracing solutions initiated at the same location $\left(3.72^{\circ} \mathrm{N}, 172.5^{\circ} \mathrm{E}\right)$ on solid-body rotation with $u=15 \mathrm{~m} \mathrm{~s}$ at the equator, with initial zonal wavenumber (a)-(d) $k=5$ and (e)-(h) $k=5+0.01 i$. Shown are (a),(e) wave latitude, (b),(f) wave amplitude, (c),(g) imaginary wavenumber parts, and (d),(h) real wavenumber parts during 15 days $(360 \mathrm{~h})$ of integration. The complex wavenumber wave behaves erratically in the vicinity of the turning latitude as the real meridional wavenumber nears zero.

$$
\beta_{M}=2 \Omega \cos ^{2} \theta / r-\frac{\partial}{\partial y}\left[\frac{1}{\cos ^{2} \theta} \frac{\partial}{\partial y}\left(\cos ^{2} \theta \bar{u}_{M}\right)\right]
$$

where $r$ is the radius of the earth; and the stationary wavenumber $K_{s}$, per Hoskins and Ambrizzi (1993),

$$
K_{s}=\left(\frac{r \beta_{M}}{u_{M}}\right)^{1 / 2}
$$

calculated for regions where $\beta_{M}>0$ and $u_{M}>0$. Regions of local maximal $\beta_{M}$ and $K_{s}$ tend to occur in the strong westerly flow associated with extratropical jets. For the JAS 300-mb atmosphere, these maxima are associated in the winter hemisphere with the southern jet and in the summer hemisphere with the North AfricanAsian and North American jets, as well as the subtropical jet over the Pacific (Fig. 10).

Among the three groupings of propagation behavior, the CRTs that propagate northward to the region of subtropical convergence and then westward remain within the easterlies over Mexico and the eastern Pacific, although a few escape northward into the westerlies and propagate eastward over the United States. (Fig. 10a). For the second grouping of CRTs that propagate to the north or northeast over the Caribbean, the rays that propagate farthest east move along the maximum of $\beta_{M}$ in the North African-Asian jet (Fig. 10b). This behavior in which the ray propagates zonally while refracting toward the jet core has also been shown to occur analogously for RRTs initiated during boreal winter (Shaman and Tziperman 2005). For the CRTs that propagate into the Southern Hemisphere, many of these rays propagate zonally once they reach westerlies near $10^{\circ} \mathrm{S}$. Some rays move farther south, become trapped in the southern jet, and propagate zonally. These behaviors indicate that the complex wavenumber Rossby wave ray tracings respond to wind patterns, gradients of absolute vorticity, and waveguides in fashion similar to real wavenumber Rossby waves.

The LBVE simulation was next performed again with the same background and forcing, but with a sponge layer at $150^{\circ} \mathrm{E}$ to the west of the forcing region, per Shaman and 
a) Real Rays

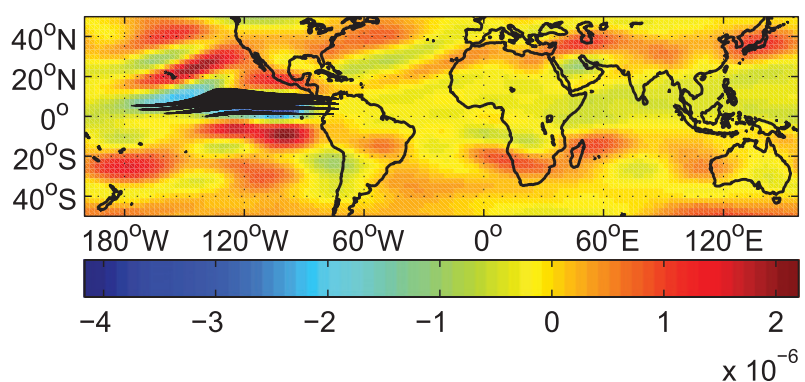

b) Complex Rays

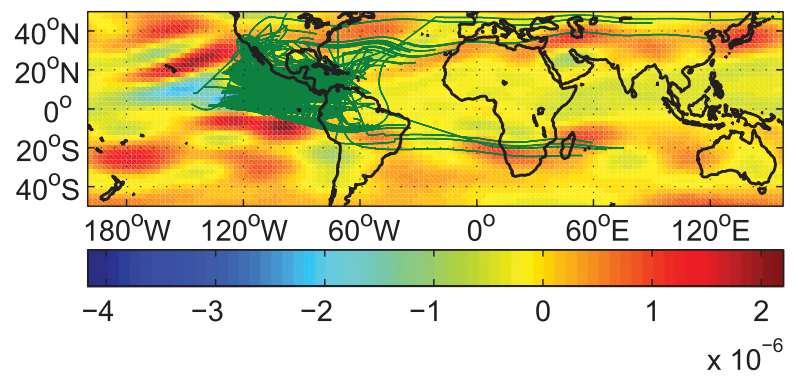

FIG. 8. Steady LBVE vorticity solution to Gaussian amplitude divergent forcing centered at $5^{\circ} \mathrm{N}, 90^{\circ} \mathrm{W}$. Divergence maximizes in center at $-3 \times 10^{-6} \mathrm{~s}^{-1}$. The background is JAS 1949-2010300-mb climatology that has been zonally smoothed to wavenumber 8 . Rossby wave ray tracings initiated throughout the forcing region with initial integer $k=4-12$ are shown: (a) all real wavenumber ray tracings and (b) all complex wavenumber ray tracings.

Tziperman (2007). This sponge layer damps out westwardpropagating Rossby waves and isolates the response due to eastward-propagating waves. This simulation reveals vorticity anomalies east of Cuba, west of Spain, and over and east of Brazil that arise within the LBVE model due to eastward-propagating disturbances (Fig. 11). These vorticity responses are consistent with CRT trajectories. More specifically, complex Rossby wave ray tracings can be seen traversing these regions (Figs. 8 and 9).

The different trajectory behaviors among the CRTs are also evident in plots of individual initiation sites (Fig. 12). Individual trajectory lines in fact depict two waves, initialized complex conjugates, one growing, one decaying, that propagate identically through space. CRTs initiated at $8.69^{\circ} \mathrm{N}, 107.5^{\circ} \mathrm{W}$ produce rays that propagate northward into the zone of subtropical convergence evident in the LBVE model solution (Fig. 12a). Complex wavenumber ray tracings initiated at $3.72^{\circ} \mathrm{N}$, $85^{\circ} \mathrm{W}$ propagate east-northeastward over the LBVE model solution anomaly in the vicinity of Cuba (Fig. 12b). These waves exhibit a greater tendency to behave erratically in trajectory as well as amplitude; however, a number of them are well behaved. Complex wavenumber ray tracings initiated at $1.24^{\circ} \mathrm{N}, 85^{\circ} \mathrm{W}$ propagate either northward

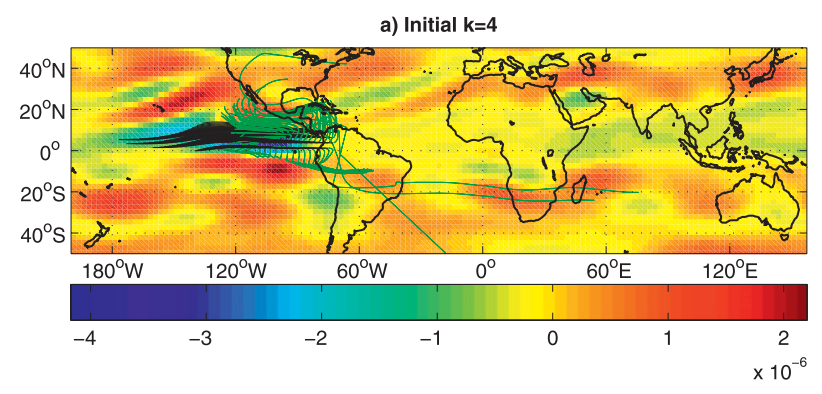

b) Initial $k=5$
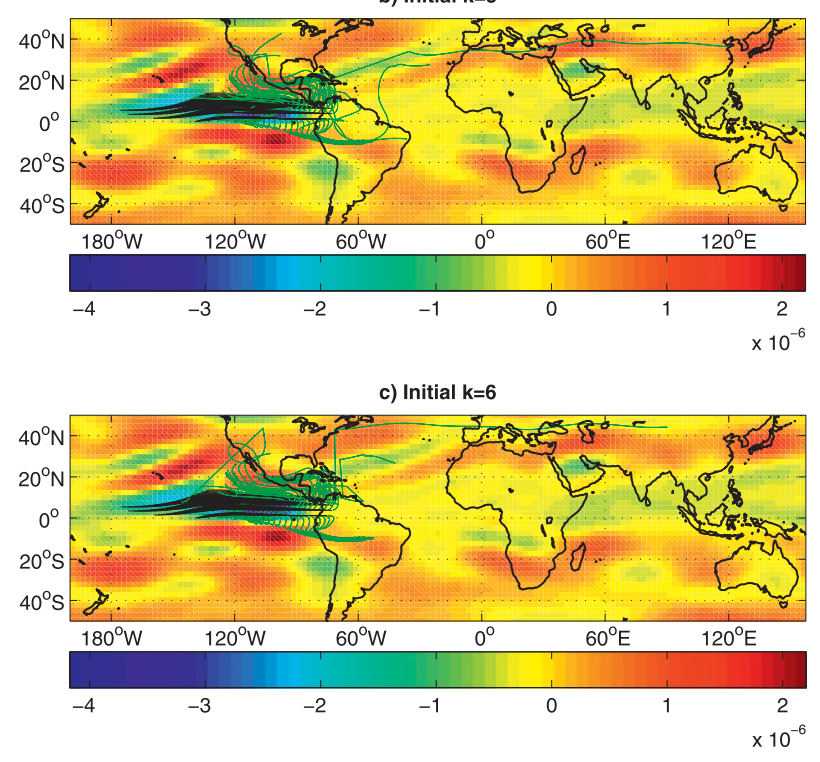

FIG. 9. As in Fig. 8, but for ray tracings initiated with initial zonal wavenumber: (a) $k=4$ only, (b) $k=5$ only, and (c) $k=6$ only. Black lines are real Rossby wave ray tracings; green lines are complex Rossby wave ray tracings.

into the zone of subtropical convergence north of the forcing region or southward and then eastward over South America (Fig. 12c).

Figures 13 and 14 present the latitude, amplitude, and wavenumber evolution over the course of 15 days integration for two of the growing complex Rossby waves shown in Fig. 12. The east-northeastward-propagating wave with initial $k=3$ (Fig. 13) traverses Cuba and nears the isolated vorticity anomaly evident in the LBVE model solution (Fig. 11) during hour 80 at which time the wave amplitude grows markedly. The traced wave peaks in amplitude around hour 100 while in the vicinity of the vorticity anomaly. During these hours of integration the wave behaves somewhat erratically as its amplitude grows precipitously: the wave jumps in latitude and wavenumbers shift or change sign. In addition, as for many of the ray tracings performed on this $2 \mathrm{D}$ JAS background, the imaginary and real wavenumber components are the same order of magnitude such that 
a)

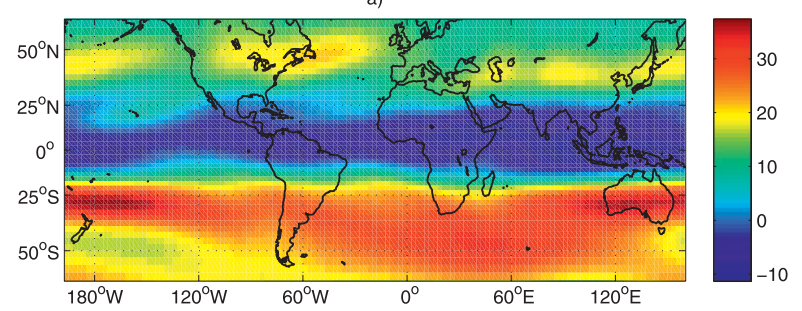

b)
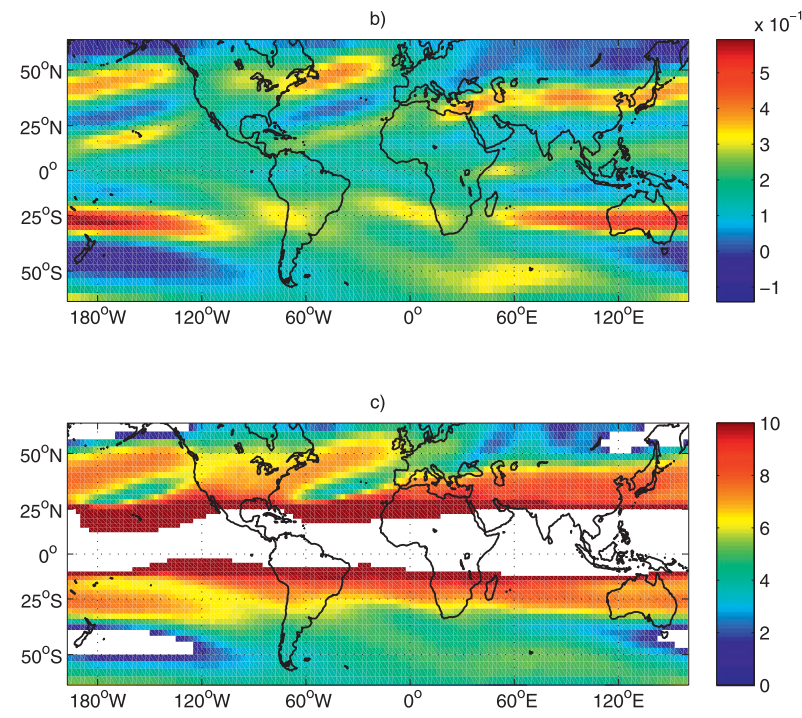

FIG. 10. Plots of 1949-2010 JAS 300-hPa NCEP-NCAR reanalysis (a) zonal wind $\left(\mathrm{m} \mathrm{s}^{-1}\right)$, (b) $\beta_{M}\left(\mathrm{~m}^{-1} \mathrm{~s}^{-1}\right)$, and (c) $K_{s}(1 / \mathrm{m})$. Values of $K_{s}$ are only shown for regions of both $u_{m}>0$ and $\beta_{M}>0$.

$\alpha \approx 1$, rather than $\alpha \ll 1$, which violates the approximation used to insure that rays remain close to the real plane. However, these complex wavenumber Rossby waves traverse the region east of Cuba and exhibit rapid growth in this area, both of which are behaviors consistent with the LBVE solution showing an isolated anomaly east of Cuba that results from an eastwardpropagating signal (Fig. 11b). In contrast, no real wavenumber Rossby waves traverse this region (Fig. 8).

While this ray-tracing amplitude and trajectory solution does not clearly corroborate the isolated anomaly finding of the LBVE model, the peak of amplitude near the anomaly is consistent with it and could perhaps be indicative of an area of Rossby wave instability. Furthermore, other waves traversing the area also grow in amplitude in this same region and qualitatively identify the anomaly region (Fig. 12).

The northward-propagating wave with initial $k=4$ (Fig. 14) grows continuously during the 15-day integration. The trajectory and amplitude of this ray tracing vary smoothly, yet for the meridional wavenumber $l_{i}$ and $l_{r}$ are the same order of magnitude over the course of the a)

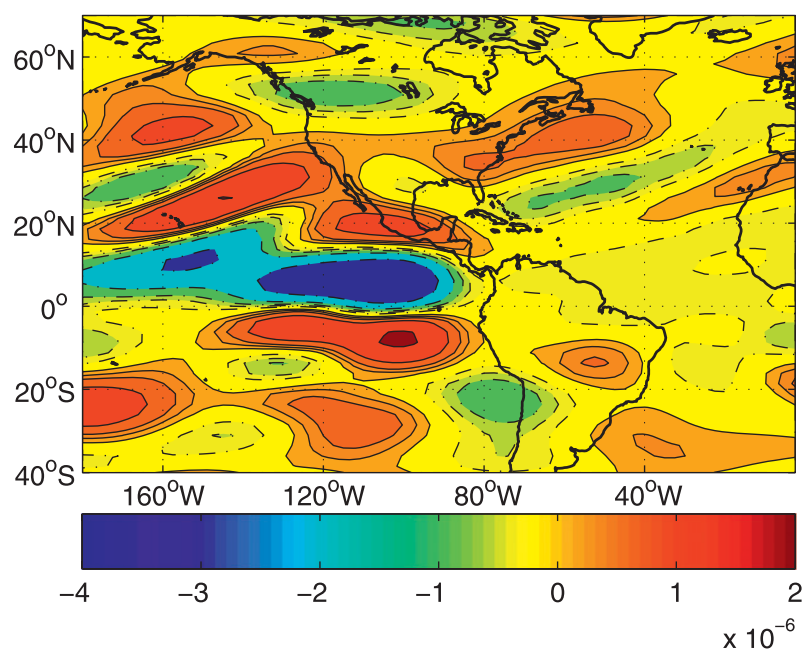

b)

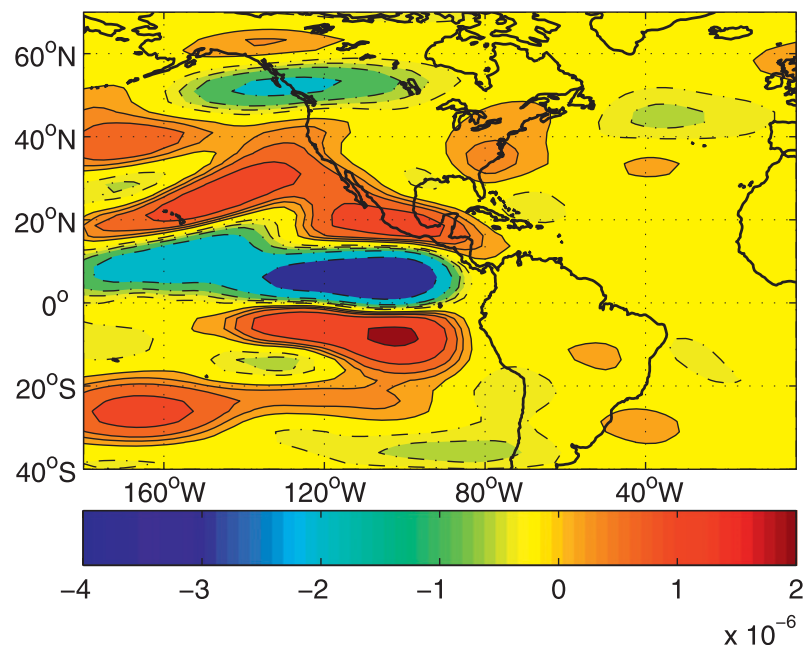

FIG. 11. LBVE vorticity solution to Gaussian amplitude divergent forcing centered at $5^{\circ} \mathrm{N}, 90^{\circ} \mathrm{W}$. Divergence maximizes in center at $-3 \times 10^{-6} \mathrm{~s}^{-1}$. The background is JAS 1949-2010 300-mb climatology that has been zonally smoothed to wavenumber 8 . (a) Full solution, as in Fig. 8. (b) As in (a), but a sponge layer has been applied at $150^{\circ} \mathrm{E}$ to preclude Rossby wave propagation westward from the Pacific basin. Contours are $\pm 0.2 \times 10^{-6}, \pm 0.4 \times 10^{-6}$, $\pm 0.6 \times 10^{-6}, \pm 1 \times 10^{-6}, \pm 2 \times 10^{-6}$, and $-4 \times 10^{-6} \mathrm{~s}^{-1}$. Negative contours are dashed.

integration. This wave propagates slowly into the region of subtropical convergence (Fig. 12a).

For all of the ray tracing presented here, the calculated amplitude does not take into account dissipative processes, nor does it define an initial wave amplitude, only relative changes in amplitude. Dissipation will decrease the wave amplitude; furthermore, the initial wave amplitude may be small. In addition, ray tracing does not account for nonlinear processes, such as wave-mean 
a)

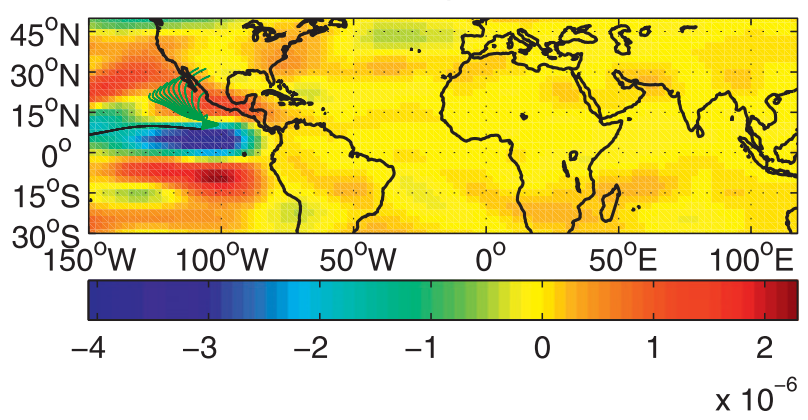

b)

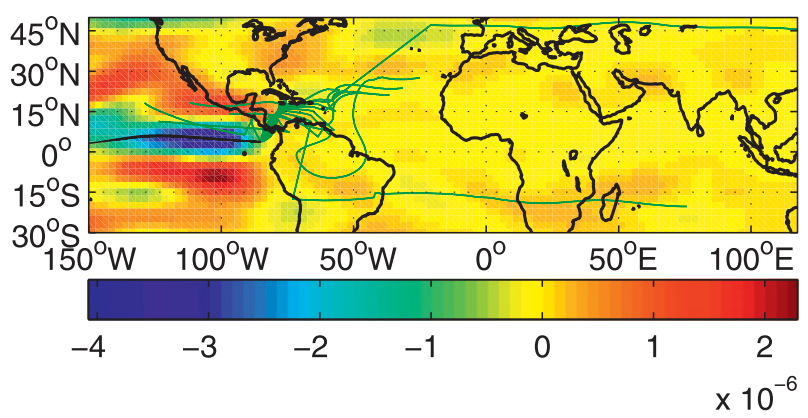

c)

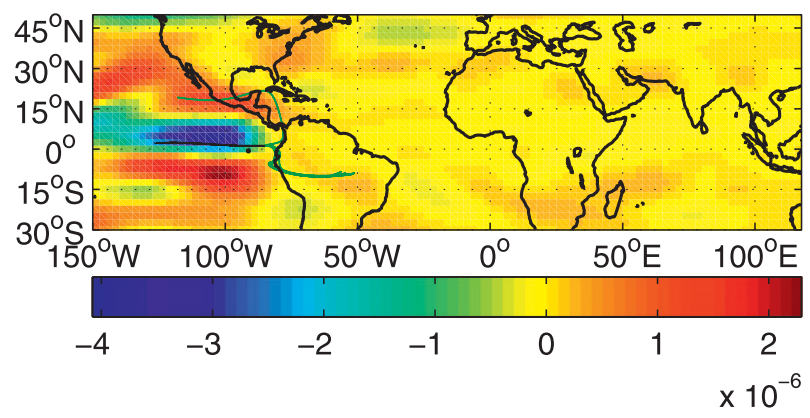

FIG. 12. Steady LBVE vorticity solution to Gaussian amplitude divergent forcing centered at $5^{\circ} \mathrm{N}, 90^{\circ} \mathrm{W}$. Divergence maximizes in center at $-3 \times 10^{-6} \mathrm{~s}^{-1}$. The background is JAS 1949-2010 300-mb climatology that has been zonally smoothed to wavenumber 8 . A sponge layer has been applied centered at $150^{\circ} \mathrm{E}$ that precludes Rossby wave propagation westward from the Pacific basin. Rossby wave ray tracings initiated at three individual sites with initial integer $k=4-12$ are shown. The sites are (a) $8.69^{\circ} \mathrm{N}, 107.5^{\circ} \mathrm{W}$; (b) $3.72^{\circ} \mathrm{N}$, $85^{\circ} \mathrm{W}$; and (c) $1.24^{\circ} \mathrm{N}, 85^{\circ} \mathrm{W}$. Black lines are real Rossby wave ray tracings; green lines are complex Rossby wave ray tracings.

flow interaction or wave breaking, which would likely dominate at large wave amplitudes. In spite of these limitations, both the trajectory and amplitude growth of the traced complex wavenumber Rossby waves are qualitatively consistent with the development of the isolated vorticity anomalies seen over Cuba and the subtropical convergence zone north of the forcing region.

For further comparison, we performed a similar experiment during boreal winter over Indonesia. The LBVE

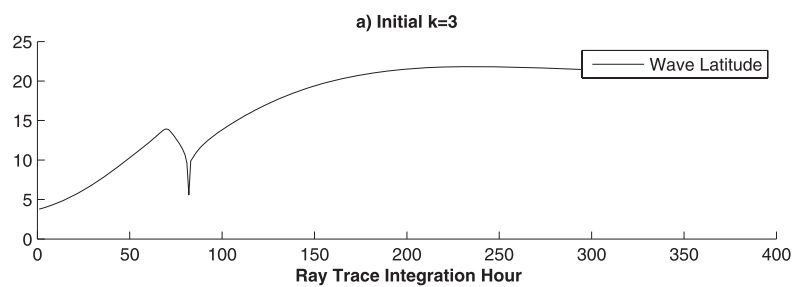

b)

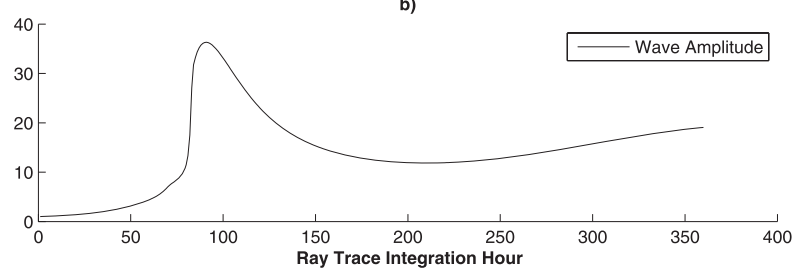

c)
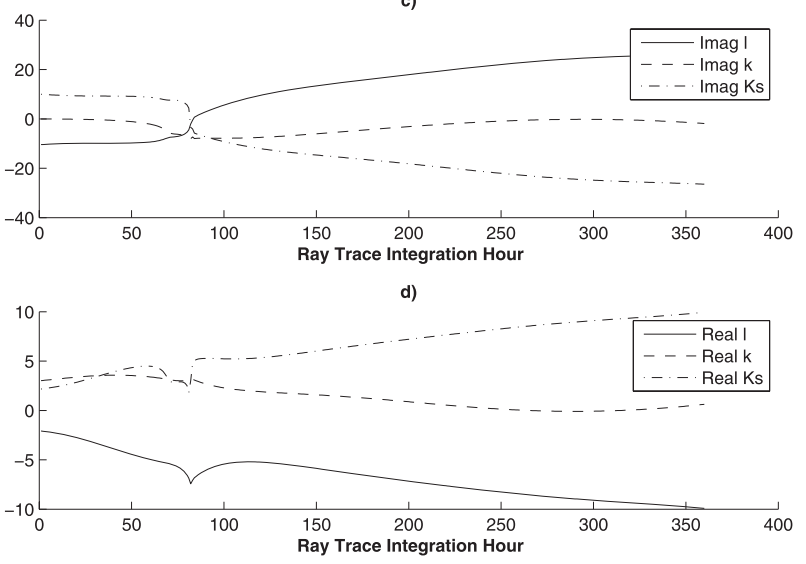

FIG. 13. Plots of (a) wave latitude, (b) wave amplitude (relative to initial value), (c) imaginary wavenumbers, and (d) real wavenumbers of the growing ray initiated with $k=3$ at $1.24^{\circ} \mathrm{N}, 85^{\circ} \mathrm{W}$.

model was linearized about 300-hPa January-March (JFM) climatology and forced with divergence centered at $5^{\circ} \mathrm{N}$, $120^{\circ} \mathrm{E}$. Rossby wave ray tracings were initiated throughout the forcing region with initial integer $k=3-6$. For this region and season, there is less distinction between the real and complex stationary barotropic Rossby wave raytracing trajectories (Fig. 15); however, real wavenumber ray tracings show a greater tendency to propagate poleward and eastward along trajectories that follow the wave train of alternating positive and negative anomalies produced by the LBVE model. The complex rays tend to remain within the tropics and also show a greater tendency to cross into the Southern Hemisphere.

The similarity of the complex and real wavenumber Rossby wave trajectories initiated over Indonesia during boreal winter (Fig. 15) differs from the tracings initiated over the eastern equatorial Pacific during boreal summer, which show a clearer divergence of complex and real wavenumber Rossby wave trajectory behaviors (Fig. 8). Differences among these two tropical environments include differences in the background winds in both the tropics and 


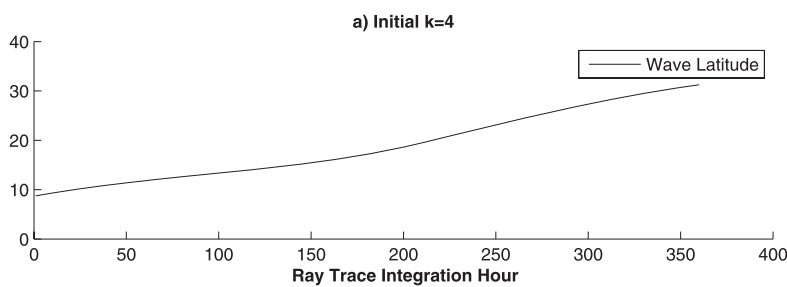

b)
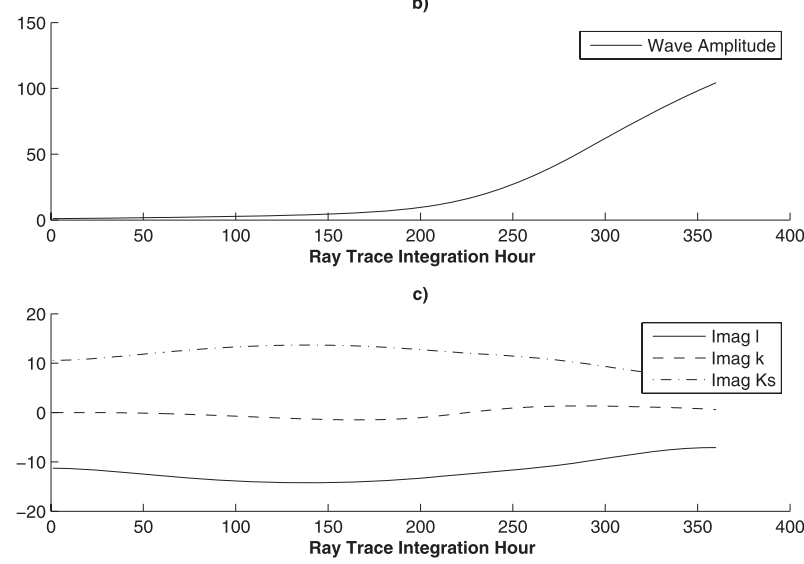

d)

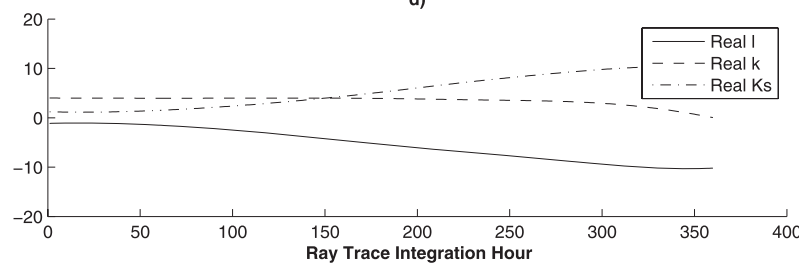

FIG. 14. As in Fig. 13, but for the growing ray initiated with $k=4$ at $8.69^{\circ} \mathrm{N}, 107.5^{\circ} \mathrm{W}$.

extratropics. These changes may account for the different forced LBVE solutions, as well as the greater dispersion of complex and real wavenumber Rossby waves initiated over the eastern equatorial Pacific during JAS. The divergence of complex versus real wavenumber ray behaviors and its dependence on the background field is a topic for future study.

\section{Comparison of complex ray-tracing methods}

As discussed above, other approaches have been proposed and developed for carrying out CRT (Brillouin 1914; Sommerfeld 1914; Censor and Gavan 1989; Sonnenschein et al. 1998; Kravtsov and Orlov 1999). Here we compare our CRT method with one alternate approach, referred to as MD93, in which wavenumbers are allowed to be complex but ray propagation is restricted to real coordinate space by considering only the real part of the group velocity (Muschietti and Dum 1993). The principal difference between the two methods is that our method neglects, via a scaling argument, only a) Real Rays

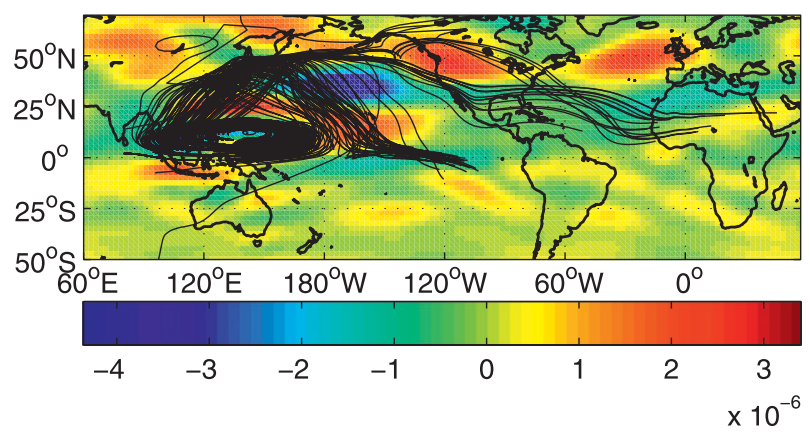

b) Complex Rays

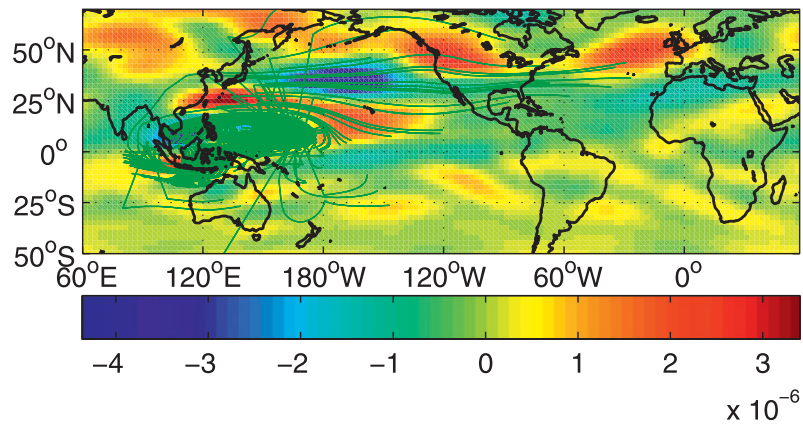

FIG. 15. Steady LBVE vorticity solution to Gaussian amplitude divergent forcing centered at $5^{\circ} \mathrm{N}, 120^{\circ} \mathrm{E}$. Divergence maximizes in center at $-3 \times 10^{-6} \mathrm{~s}^{-1}$. The background is JFM 1949-2010 300-mb climatology that has been zonally smoothed to wavenumber 8. Rossby wave ray tracings initiated throughout the forcing region with initial integer $k=3-6$ are shown, for (a) real wavenumber ray tracings and (b) complex wavenumber ray tracings.

the last term of Eq. (2.5a), whereas the alternate approach neglects the last term of both Eqs. (2.5a) and (2.5b).

CRT performed using the MD93 approach was applied to the NCEP-NCAR reanalysis JAS 1949-2010 300-hPa relative vorticity climatology and initiated at forcing sites within $0^{\circ}-10^{\circ} \mathrm{N}$ and $110^{\circ}-70^{\circ} \mathrm{W}$ for initial $k=4-12$ (Fig. 16). As a whole, the results are qualitatively similar to our perturbation approach. Complex ray tracings with the MD93 approach are also distinct from RRT solutions, which remain within the equatorial waveguide (Fig. 8). As in our perturbation approach, the MD93 CRTs fall into three general groupings: 1) propagation northward to the region of subtropical convergence over and west of Mexico; 2) propagation over the Caribbean, Cuba, and in some instances into the North African-Asian jet; and 3) propagation southward to about $10^{\circ} \mathrm{S}$ and then eastward.

Based on these findings it is difficult to identify one CRT approach as superior. Both methods produce similar gross descriptions of the complex wavenumber Rossby wave response to forcing over the eastern equatorial 


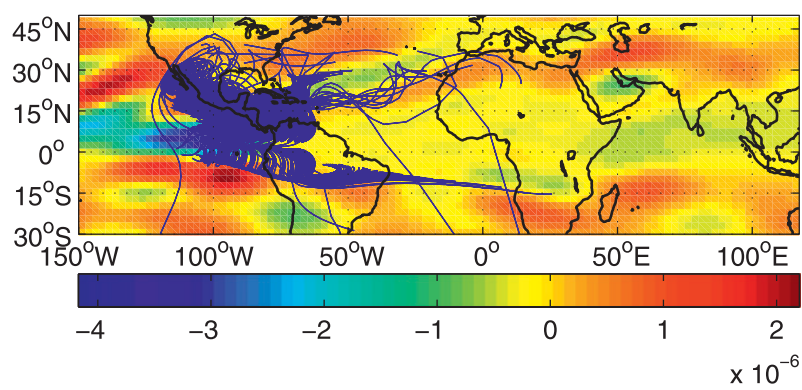

FIG. 16. As in Fig. 8, but only for complex wavenumber Rossby wave ray tracings performed per Muschietti and Dum (1993) in which wavenumbers are complex but ray propagation is restricted to the real coordinate space by considering only the real part of the group velocity.

Pacific during boreal summer. Both have rays that traverse the isolated anomalies east of Cuba, west of Spain, and over and east of Brazil that arise within the LBVE model due to eastward-propagating disturbances (Fig. 11b). Both methods produce rays that grow in the vicinity of these isolated anomalies (not shown). Both methods produce some rays with erratic, aphysical behavior.

Indeed, the MD93 approach is simpler to apply, and these findings suggest that this method can also be justifiably used for tracing Rossby waves. However, individual ray trajectories differ between the two methods: that is, the same initial complex ray follows a different path depending on the tracing method used (Figs. 12 and 17). The perturbation method we have introduced includes a scaling formalism that accounts for an additional term, $u_{g i}\left(\partial k_{r} / \partial x\right)$ [Eq. (2.5b)], that is neglected when the imaginary group velocity is dropped in the MD93 approach. For ray tracings where $\alpha \ll 1$, our method should be more accurate, although admittedly for many of the ray tracings this scaling does not hold. In such instances, it is difficult to determine which tracing method is superior. Still, overall, both CRT methods produce a family of rays that depict responses seen in simulations with the LBVE model and not produced by RRT.

\section{Discussion}

Here we have shown that complex wavenumber stationary barotropic Rossby wave ray tracing can be used to track the amplitude and trajectory of simultaneously propagating and growing or decaying forced disturbances. Ray-tracing amplitude solutions for these waves match exact solutions in a simple, idealized setting. Trajectories of these complex wavenumber Rossby wave ray tracings match forced solutions of a barotropic vorticity equation model linearized about a state of solid-body rotation, as do real wavenumber Rossby wave ray-tracing trajectories. a)

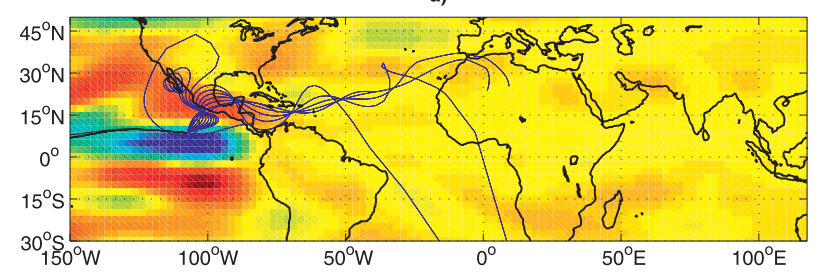

b)

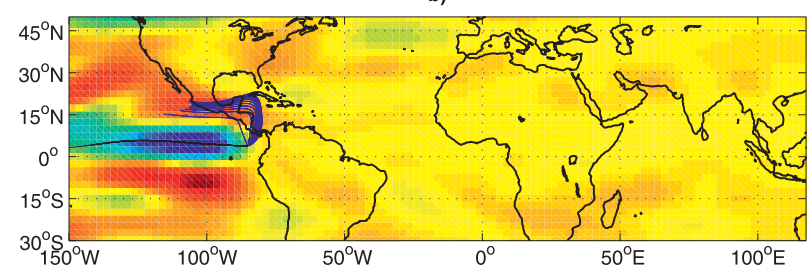

c)

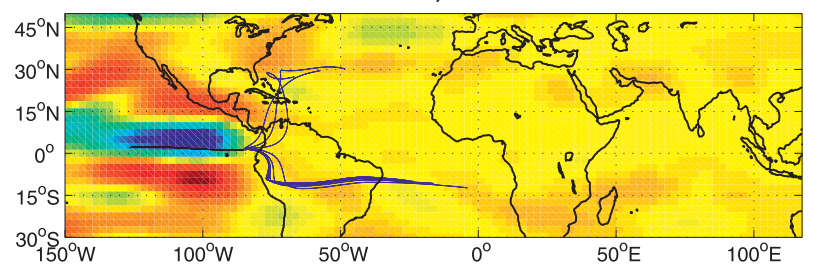

FIG. 17. As in Fig. 12, but only for Rossby wave ray tracings performed per Muschietti and Dum (1993) in which wavenumbers may be complex but ray propagation is restricted to real coordinate space by considering only the real part of the group velocity. Black lines are real Rossby wave ray tracings; blue lines are complex Rossby wave ray tracings.

Complex wavenumber Rossby wave ray tracing in a realistic zonally asymmetric atmosphere produces trajectories that are distinct from real wavenumber Rossby wave ray tracings and coincident with vorticity anomalies generated in similarly forced solutions of the LBVE model. Thus, it appears that inclusion of complex wavenumber stationary barotropic Rossby waves in addition to the real wavenumber ray tracings provides a more complete description of the LBVE model solution. The amplitudes of these distinct complex wavenumber wave trajectories appear, on a qualitative level, to amplify in regions where the LBVE model vorticity solutions maximize.

Care must be taken when using this ray-tracing approach. Many of the integrations performed violate the assumption that $\alpha \ll 1$. In some instances this violation is associated with unusual and nonphysical behaviors. This erratic behavior of some of the complex waves may arise from a violation of WKBJ assumptions near a turning latitude (Karoly and Hoskins 1982), a violation of the assumption that $\alpha \ll 1$, or both. It is noteworthy that for solid-body rotation no real wavenumber Rossby wave ray tracings were found to behave in this fashion near turning latitudes (Fig. 6). Consequently, the erratic behavior of some complex wavenumber ray tracings at and beyond 
a turning latitude likely arises from violation of the assumption that $\alpha \ll 1$ or from violation of both WKBJ and $\alpha \ll 1$.

In spite of this erratic behavior from some complex wavenumber Rossby wave ray tracings, many of the other complex wavenumber wave trajectories propagate without large wavenumber changes over a single time step or radical shifts in direction or location. This finding suggests that the technique presented here can provide useful insight even when formal scaling assumptions are violated. Indeed, ray tracing of real wavenumber waves often violates WKBJ assumptions yet still proves informative (Karoly and Hoskins 1982).

CRT has been performed here for an inviscid atmosphere; however, complex wavenumbers can be introduced if the medium is unstable or dissipative. Indeed, inclusion of dissipative processes might be warranted. While our Rossby wave dispersion relation (3.2) is inviscid, the LBVE model solutions to which the CRT is compared include damping and diffusion (appendix C). To better reflect the LBVE model, we could include dissipation in the barotropic vorticity equation for the perturbation streamfunction,

$$
\frac{\partial \nabla^{2} \psi^{\prime}}{\partial t}+J\left(\bar{\psi}, \nabla^{2} \psi^{\prime}\right)+J\left(\psi^{\prime}, \nabla^{2} \bar{\psi}+f\right)+\nu \nabla^{2} \psi^{\prime}=0,
$$

where $\nu$ is the Rayleigh damping coefficient. For a plane wave of the form $\psi=A \exp [i(k x+l y-\omega t)]$ this yields the dispersion relation

$$
\omega=\bar{u} k+\bar{v} l+\frac{l \partial \bar{q} / \partial x-k \partial \bar{q} / \partial y}{k^{2}+l^{2}}-i \nu .
$$

Typically, damping is ignored and the atmosphere is treated as inviscid for the purpose of Rossby wave ray tracing (Hoskins and Karoly 1981; Karoly 1983; Li and Nathan 1997); however, Eq. (8.1) is a valid Rossby wave expression that would produce complex wavenumber Rossby waves, which would undergo weak decay while propagating due to this damping. This framework is analogous to the one addressed by Muschietti and Dum (1993) and Censor and Gavan (1989), who both consider complex wavenumbers and group velocities for systems with complex dispersion relations. Our use of an inviscid Rossby wave dispersion relation (3.2) allows for both real and complex wavenumber waves. Explicit decay due to damping is not represented, but rather the more dynamically interesting cases of growth or decay due to spatial instability are depicted.

Rossby wave ray tracing also need not be restricted to stationary waves. Tropically forced, low-frequency, nonstationary Rossby waves have been examined in prior studies ( $\mathrm{Li}$ and Nathan 1997) to explore the planetaryscale response to time-varying forcing. It has been found that for the same forcing location and initial zonal wavenumber but different wave frequencies, the trajectories of these waves diverge. In fact, by merely changing the Rossby wave frequency $\omega$, the initial meridional wavenumber solutions changes, and in some instances can change from real to complex. Consequently CRT of low-frequency, nonstationary Rossby waves will provide a fuller representation of the planetary scale response to such low-frequency time-varying forcing than RRT alone. Such investigation, which is left for a future work, may provide a more complete picture of atmospheric response to periodic forcing at time scales that are subseasonal but beyond the limits of numerical weather prediction.

Our perturbation method for CRT is appealing due to the relative simplicity of the approach, the scaling formalism that retains an appropriate additional term in the ray-tracing equation (provided $\alpha \ll 1$ ), and the fact that CRT solutions replicate LBVE model behavior not captured by RRT. However, the still simpler MD93 approach produces qualitatively similar Rossby wave raytracing results in a zonally asymmetric two-dimensional atmosphere. It is difficult to assess quantitatively which of these methods is indeed more accurate. Future comparison of these two approaches might focus on cataloging the ability of CRT approaches to capture unusual phenomena. Indeed, CRT of Rossby waves may provide insight into energy accumulation (e.g., Webster and Chang 1988), caustics in the atmosphere, critical layers, and spatial instabilities, such as in frontal zones.

CRT of the full Eqs. (2.5) into complex space might also be attempted and used for comparison. Such a procedure, although more cumbersome, might use analytic continuation to project the basic state into complex space (e.g., using Chebyshev polynomials), perform ray tracing in complex space, and then project the solutions back to real space.

The LBVE model used in this study provides a highly simplified depiction of large-scale atmospheric response and does not include nonlinear, baroclinic, or moist processes. Furthermore, by restricting LBVE model forcing to the immediate region of equatorial divergence [i.e., $-(f+\zeta) D$ ] rather than the full Rossby wave source, as described by Sardeshmukh and Hoskins (1988), the model depiction is further removed from the response of the true atmosphere, or even a GCM. However, use of only the simple $-(f+\zeta) D$ forcing provides a useful means for explicit comparison of CRT and model solutions; that is, rays and the LBVE model are only forced in a single region and secondary forcing regions that can arise from vorticity advection of the divergent flow are not depicted in either the LBVE model or CRT. 
Still, a truer representation of the actual atmospheric response to equatorial heating would allow for vorticity advection by the divergent flow and downstream Rossby wave generation (Sardeshmukh and Hoskins 1988). A useful follow-up study and application of CRT would be to explore whether the inclusion of complex wavenumber Rossby wave ray tracings better represents the trajectories of such secondarily forced Rossby waves from downstream regions of initiation than just standard real wavenumber Rossby wave ray tracing alone. Such a study would use ray tracing to understand better the complete large-scale barotropic atmospheric response to thermal or orographic forcing.

A key question to be examined further in future studies is whether complex rays describe real-world, physical phenomena not described through RRT. The evidence from this study, and prior work in other disciplines, suggests that CRT can represent wave patterns not captured by RRT alone. Complex wavenumber plane wave solutions simultaneously propagate and either grow or decay, unlike real wavenumber solutions, which only propagate, and purely imaginary wavenumber solutions, which only grow or decay.

In the work presented here, for some CRT, the imaginary components of the zonal and meridional wavenumbers of these complex Rossby waves could have offsetting effects on wave amplitude. That is, if the signs of the imaginary components of the two wavenumbers, $k_{i}$ and $l_{i}$, are opposite, one will act to increase wave amplitude while the other acts to decrease wave amplitude. The combined effect is that the amplitude of the wave may not grow or decay rapidly and thus may produce a signal like a real wavenumber Rossby wave. Dickinson and Clare (1973) noted that slowly decaying complex wavenumber Rossby waves could persist for some time in the atmosphere and ultimately extract momentum from the mean flow at considerable distances from their initial generation.

Future work is needed to determine the full utility of this methodology and complex wavenumber Rossby wave ray tracing in general. In particular, the factors controlling the dispersion of complex and real wavenumber ray trajectories need to be further investigated in order to determine the conditions in which these two wavenumber types characterize distinct behaviors.

Acknowledgments. We thank Ian Eisenman and Michael Brenner for helpful discussions. This work was supported by NSF Grants AGS-1205043 (JS), OCE0424516 (RMS), and ATM-0917468 (ET).

\section{APPENDIX A}

\section{Wavenumber Gradient Equation Components for Two-Dimensional Barotropic Rossby Waves}

To construct Eqs. (2.12)-(2.15) for two-dimensional barotropic Rossby waves, we need the following additional expressions:

$$
\begin{aligned}
\left.\frac{\partial^{2} \omega}{\partial k \partial x}\right|_{r} & =\operatorname{Re}\left[\frac{\partial \bar{u}_{M}}{\partial x}+\frac{\left(k^{2}-l^{2}\right) \partial^{2} \bar{q} / \partial y \partial x-2 k l \partial^{2} \bar{q} / \partial x^{2}}{\left(k^{2}+l^{2}\right)^{2}}\right], \\
\left.\frac{\partial^{2} \omega}{\partial k \partial y}\right|_{r} & =\operatorname{Re}\left[\frac{\partial \bar{u}_{M}}{\partial y}+\frac{\left(k^{2}-l^{2}\right) \partial^{2} \bar{q} / \partial y^{2}-2 k l \partial^{2} \bar{q} / \partial x \partial y}{\left(k^{2}+l^{2}\right)^{2}}\right], \\
\left.\frac{\partial^{2} \omega}{\partial k^{2}}\right|_{r} & =\operatorname{Re}\left\{\frac{\left(k^{2}+l^{2}\right)(2 k \partial \bar{q} / \partial y-2 l \partial \bar{q} / \partial x)-\left[\left(k^{2}-l^{2}\right) \partial \bar{q} / \partial y-2 k l \partial \bar{q} / \partial x\right] 4 k}{\left(k^{2}+l^{2}\right)^{3}}\right\}, \\
\left.\frac{\partial^{2} \omega}{\partial l^{2}}\right|_{r} & =\operatorname{Re}\left\{\frac{\left(k^{2}+l^{2}\right)(2 k \partial \bar{q} / \partial y-2 l \partial \bar{q} / \partial x)-\left[2 k l \partial \bar{q} / \partial y+\left(k^{2}-l^{2}\right) \partial \bar{q} / \partial x\right] 4 l}{\left(k^{2}+l^{2}\right)^{3}}\right\}, \\
\left.\frac{\partial^{2} \omega}{\partial l \partial k}\right|_{r} & =\operatorname{Re}\left\{\frac{\left(k^{2}+l^{2}\right)(-2 l \partial \bar{q} / \partial y-2 k \partial \bar{q} / \partial x)-\left[\left(k^{2}-l^{2}\right) \partial \bar{q} / \partial y-2 k l \partial \bar{q} / \partial x\right] 4 l}{\left(k^{2}+l^{2}\right)^{3}}\right\}, \\
\left.\frac{\partial^{2} \omega}{\partial l \partial x}\right|_{r} & =\operatorname{Re}\left[\frac{\left.\partial \bar{v}_{M}+\frac{2 k l \partial^{2} \bar{q} / \partial x \partial y+\left(k^{2}-l^{2}\right) \partial^{2} \bar{q} / \partial x^{2}}{\partial x}\right],}{\left(k^{2}+l^{2}\right)^{2}}\right.
\end{aligned}
$$




$$
\begin{aligned}
\left.\frac{\partial^{2} \omega}{\partial l \partial y}\right|_{r} & =\operatorname{Re}\left[\frac{\partial \bar{v}_{M}}{\partial y}+\frac{2 k l \partial^{2} \bar{q} / \partial y^{2}+\left(k^{2}-l^{2}\right) \partial^{2} \bar{q} / \partial x \partial y}{\left(k^{2}+l^{2}\right)^{2}}\right], \\
\left.\frac{\partial^{2} \omega}{\partial x^{2}}\right|_{r} & =\operatorname{Re}\left[k \frac{\partial^{2} \bar{u}_{M}}{\partial x^{2}}+l \frac{\partial^{2} \bar{v}_{M}}{\partial x^{2}}+\frac{l \partial^{3} \bar{q} / \partial x^{3}-k \partial^{3} \bar{q} / \partial x^{2} \partial y}{\left(k^{2}+l^{2}\right)}\right], \\
\left.\frac{\partial^{2} \omega}{\partial y^{2}}\right|_{r} & =\operatorname{Re}\left[k \frac{\partial^{2} \bar{u}_{M}}{\partial y^{2}}+l \frac{\partial^{2} \bar{v}_{M}}{\partial y^{2}}+\frac{l \partial^{3} \bar{q} / \partial x \partial y^{2}-k \partial^{3} \bar{q} / \partial y^{3}}{\left(k^{2}+l^{2}\right)}\right], \\
\left.\frac{\partial^{2} \omega}{\partial y \partial x}\right|_{r} & =\operatorname{Re}\left[k \frac{\partial^{2} \bar{u}_{M}}{\partial y \partial x}+l \frac{\partial^{2} \bar{v}_{M}}{\partial y \partial x}+\frac{l \partial^{3} \bar{q} / \partial y \partial x^{2}-k \partial^{3} \bar{q} / \partial y^{2} \partial x}{\left(k^{2}+l^{2}\right)}\right] .
\end{aligned}
$$

These expressions are all derived from Eq. (3.2) and can be used to construct the ray-tracing equations for $\partial k_{r} / \partial x, \partial k_{r} / \partial y, \partial l_{r} / \partial x$, and $\partial l_{r} / \partial y$, and thus also Eqs. (2.10c) and (2.10d) for $k_{i}$ and $l_{i}$.

\section{APPENDIX B}

\section{Solution to the Analytic System}

The four waves satisfy two boundary conditions at $y=$ 0 , discontinuity matching of the quasigeostrophic potential vorticity equation and continuity of pressure across the boundary (i.e., $\psi$ is continuous). At $y=Y$, we have only three waves to consider, the two northwardpropagating region II complex waves and the region III northward-propagating real wave. These three waves must satisfy the same two boundary conditions at $y=Y$.

The quasigeostrophic potential vorticity equation with $v_{M}=0$ is

$$
\left(\partial_{t}+u_{M} \partial_{x}\right) \nabla^{2} \psi+\beta \psi_{x}+\bar{q}_{x} \psi_{y}=0
$$

Discontinuities in the effective $\beta$ occur at $y=0$ at $y=$ $Y$, which require use of a jump condition. To get this we integrate Eq. (B.1) from $-\epsilon$ to $+\epsilon$ on either side of these bounds and equate the resulting equation with zero (Wang and Fyfe 2000). All terms on the lhs but two vanish as $\epsilon \rightarrow 0$, yielding

$$
\left.\left(\omega+u_{M} k\right) \psi_{y}\right|_{-\epsilon} ^{+\epsilon}+\left.\bar{q}_{x} \psi\right|_{-\epsilon} ^{+\epsilon}=0,
$$

which is our discontinuity matching condition [Eqs. (4.2b) and (4.2d)].

We consider stationary plane waves solutions of the form $\psi=A \operatorname{expi}(k x+l y)$ for the system described in Eqs. (4.2). This yields the following equations:

$$
\begin{aligned}
1+A_{\mathrm{I} s} & =A_{\mathrm{II} g}+A_{\mathrm{II} d}, \\
l_{\mathrm{I} n}+l_{\mathrm{I} s} A_{\mathrm{I} s} & =l_{\mathrm{II} g} A_{\mathrm{IIg}}+l_{\mathrm{II} d} A_{\mathrm{II} d}, \\
A_{\mathrm{III}} & =A_{\mathrm{II} g} e^{i l_{\mathrm{IIg}} Y}+A_{\mathrm{II} d} e^{i l_{\mathrm{II} d} Y}, \\
l_{\mathrm{III}} A_{\mathrm{III}} & =l_{\mathrm{II} g} A_{\mathrm{II} g} e^{i l_{\mathrm{IIg}} Y}+l_{\mathrm{II} d} A_{\mathrm{II} d} e^{i l_{\mathrm{IId}} Y} .
\end{aligned}
$$

For the solutions for a prescribed background $\left(u_{M}, v_{M}\right.$, $\left.\beta, \beta_{T}, \partial \bar{q} / \partial x\right)$ and initial zonal wavenumber and wave frequency, the meridional wavenumber of each wave $l_{\mathrm{I} n}, l_{\mathrm{I} s}, l_{\mathrm{II} g}, l_{\mathrm{II} d}$, and $l_{\mathrm{III}}$ is found using Eq. (3.2). Solving for the amplitudes yields the following solutions:

$$
\begin{aligned}
& A_{\mathrm{II} d}=\frac{l_{\mathrm{I} n}-l_{\mathrm{Is}}}{\left[\left(l_{\mathrm{Is}}-l_{\mathrm{II} g}\right)\left(l_{\mathrm{III}}-l_{\mathrm{II} d}\right) /\left(l_{\mathrm{III}}-l_{\mathrm{IIg} g}\right)\right] e^{i\left(l_{\mathrm{II} d}-l_{\mathrm{II} g}\right) Y}+\left(l_{\mathrm{II} d}-l_{\mathrm{Is}}\right)}, \\
& A_{\mathrm{II} g}=\frac{A_{\mathrm{II} d}\left(l_{\mathrm{II} d}-l_{\mathrm{III}}\right)-\left(l_{\mathrm{I} n}+l_{\mathrm{II} s}\right)}{l_{\mathrm{I} s}-l_{\mathrm{II} g}}, \\
& A_{\mathrm{III}}=A_{\mathrm{IIg}} e^{i\left(l_{\mathrm{II}}-l_{\mathrm{III}}\right) Y}+A_{\mathrm{II} d} e^{i\left(l_{\mathrm{II} d}-l_{\mathrm{III}}\right) Y}, \\
& A_{\mathrm{I} s}=A_{\mathrm{II} g}+A_{\mathrm{II} d}-1 .
\end{aligned}
$$


translocation while traversing the region II domain in the meridional direction. An analytic solution of Rossby wave zonal translocation within region II is determined based on the ratio of the zonal and meridional wavenumbers and region II extent, $Y$ for comparison.

\section{APPENDIX C}

\section{Solutions of the Linearized Barotropic Vorticity Equation}

Forced solutions of the linearized barotropic vorticity equation were found following the solution method of Branstator (1983). The barotropic vorticity equation was first linearized about a low-pass filter (zonal wavenumbers $0-8$ ) around a background streamfunction field (e.g., solid-body rotation). The equation to be solved is then

$J\left(\bar{\psi}, \nabla^{2} \psi^{\prime}\right)+J\left(\psi^{\prime}, \nabla^{2} \bar{\psi}+f\right)+\nu \nabla^{2} \psi^{\prime}+\kappa \nabla^{4} \nabla^{2} \psi^{\prime}=R$,

where $f$ is the Coriolis force, $\nu$ is the Rayleigh coefficient, $\kappa$ is a diffusion coefficient, $R$ is a forcing function,

$$
J(A, B)=\frac{1}{r^{2}}\left(\frac{\partial A}{\partial \lambda} \frac{\partial B}{\partial \mu}-\frac{\partial A}{\partial \mu} \frac{\partial B}{\partial \lambda}\right)
$$

is the Jacobian, $\lambda$ is longitude, $\mu=\sin (\varphi), \varphi$ is latitude, and $r$ is the earth's radius. The overbars indicate the seasonal time-mean flow (the basic state); primes signify the perturbation flow to be solved; $\nu$ was set to $1.57 \times$ $10^{-6} \mathrm{~s}^{-1}$ [an $e$-folding time of $1 /(7$ days $)$ ], and $\kappa$ was set to $2.34 \times 10^{16} \mathrm{~m}^{4} \mathrm{~s}^{-1}$. The anomaly forcing was specified as $R=-\left(f+\nabla^{2} \bar{\psi}\right) D$, where $D$, forcing divergence, is set to $3 \times 10^{-6} \mathrm{~s}^{-1}$. Equation (C.1) was solved using spherical harmonics and triangular 24 truncation (T24).

\section{REFERENCES}

Bender, C. M., and S. A. Orszag, 1978: Advanced Mathematical Methods for Scientists and Engineers. McGraw-Hill, 593 pp.

Branstator, G., 1983: Horizontal energy propagation in a barotropic atmosphere with meridional and zonal structure. J. Atmos. Sci., 40, 1689-1708.

Bretherton, F. P., and C. J. R. Garrett, 1969: Wavetrains in inhomogeneous moving media. Proc. Roy. Soc. London, 302A, $529-554$.

Brillouin, L., 1914: Uber die fortpflanzung des lichtes in diesperdierenden medien. Ann. Phys., 44, 203-240. [English translation available in Brillouin, L., 1960: About the propagation of light in dispersive media. Wave Propagation and Group Velocity, Pure and Applied Physics, Vol. 8, Academic Press, 43-83.]

Budden, K. G., and P. D. Terry, 1970: Radio ray tracing in complex space. Proc. Roy. Soc. London, 321A, 275-301.
Buehler, O., 2009: Waves and Mean Flows. Cambridge University Press, 341 pp.

Censor, D., and J. J. Gavan, 1989: Wave packets, group velocities, and rays in lossy media revisited. IEEE Trans. Electromagn. Compat., 31, 262-272.

Connor, K. A., and L. B. Felsen, 1974: Complex space-time rays and their application to pulse propagation in lossy dispersive media. Proc. IEEE, 62, 1586-1598.

Dickinson, R. E., and F. J. Clare, 1973: Numerical study of the unstable modes of a hyperbolic-tangent barotropic shear flow. J. Atmos. Sci., 30, 1035-1049.

Hogg, N. G., 1988: Stochastic wave radiation by the Gulf Stream. J. Phys. Oceanogr., 18, 1687-1701.

Hoskins, B. J., and K. Karoly, 1981: The steady linear response of a spherical atmosphere to thermal and orographic forcing. J. Atmos. Sci., 38, 1179-1196.

— , and T. Ambrizzi, 1993: Rossby wave propagation on a realistic longitudinally varying flow. J. Atmos. Sci., 50, 1661-1671.

Jones, R. M., 1970: Ray theory for lossy media. Radio Sci., 5, 793-801.

Kalnay, E., and Coauthors, 1996: The NCEP/NCAR 40-Year Reanalysis Project. Bull. Amer. Meteor. Soc., 77, 437-471.

Karoly, D., 1983: Rossby wave propagation in a barotropic atmosphere. Dyn. Atmos. Oceans, 7, 111-125.

, and B. Hoskins, 1982: Three-dimensional propagation of planetary waves. J. Meteor. Soc. Japan, 60, 109-122.

Keller, J. B., and F. C. Karal Jr., 1960: Surface wave excitation and propagation. J. Appl. Phys., 31, 1039-1046.

— Gaussian beams. J. Opt. Soc. Amer., 61, 40-43.

Kravtsov, Y. A., 2005: Geometrical Optics in Engineering Physics. 1st ed. Alpha Science International Ltd., 355 pp.

- , and Y. I. Orlov, 1999: Caustics, Catastrophes and Wave Fields. 2nd ed. Springer-Verlag, 216 pp.

_ , G. W. Forbes, and A. A. Asatryan, 1999: Theory and applications of complex rays. Prog. Opt., 39, 3-62.

Li, L., and T. R. Nathan, 1994: The global atmospheric response to low-frequency tropical forcing: Zonally averaged basic states. J. Atmos. Sci., 51, 3412-3426.

—, and — 1997: Effects of low-frequency tropical forcing on intraseasonal tropical-extratropical interactions. J. Atmos. Sci., 54, 332-346.

Lighthill, J., 1978: Waves in Fluids. Cambridge University Press, $504 \mathrm{pp}$.

Merkine, L.-O., 1977: Convective and absolute instability of baroclinic eddies. Geophys. Astrophys. Fluid Dyn., 9, 129-157.

— of localized unstable baroclinic disturbances. Geophys. Astrophys. Fluid Dyn., 16, 175-206.

Muschietti, L., and C. T. Dum, 1993: Real group velocity in a medium with dissipation. Phys. Fluids, 5B, 1383-1397.

Poli, E., G. V. Pereverzev, A. G. Peeters, and M. Bornatici, 2001: EC beam tracing in fusion plasmas. Fusion Eng. Des., 53, 9-21.

Sardeshmukh, P. D., and B. J. Hoskins, 1988: The generation of global rotational flow by steady idealized tropical divergence. J. Atmos. Sci., 45, 1228-1251.

Shaman, J., and E. Tziperman, 2005: The effect of ENSO on Tibetan Plateau snow depth: A stationary wave teleconnection mechanism and implications for the South Asian monsoons. J. Climate, 18, 2067-2079.

— and - 2007: The summertime ENSO-North African-Asian jet teleconnection and implications for the Indian monsoons. Geophys. Res. Lett., 34, L11702, doi:10.1029/2006GL029143. 
Sobel, A. H., and C. S. Bretherton, 1999: Development of synopticscale disturbance over the summertime tropical northwest Pacific. J. Atmos. Sci., 56, 3106-3127.

Sommerfeld, A., 1914: Uber die fortpflanzung des lichtes in diesperdierenden medien. Annalen der Physik, 44, 177-202. [English translation available in Brillouin, L., 1960: About the propagation of light in dispersive media. Wave Propagation and Group Velocity, Pure and Applied Physics, Vol. 8, Academic Press, 17-42.]

Sonnenschein, E., I. Rutkevich, and D. Censor, 1998: Wave packets, rays, and the role of real group velocity in absorbing media. Phys. Rev. E, 57, 1005-1016.
Wang, X. H., and J. Fyfe, 2000: Onset of edge wave breaking in an idealized model of the polar stratospheric vortex. J. Atmos. Sci., 57, 956-966.

Wang, Y., K. Yamazaki, and Y. Fujiyoshi, 2007: The interaction between two separate propagations of Rossby waves. Mon. Wea. Rev., 135, 3521-3540.

Webster, P. J., and H.-R. Chang, 1988: Equatorial energy accumulation and emanation regions: Impacts of a zonally varying basic state. J. Atmos. Sci., 45, 803-829.

Whitham, G. B., 1974: Linear and Nonlinear Waves. John Wiley and Sons, $636 \mathrm{pp}$. 